\title{
Investigations of sliding-induced solute transport in articular cartilage
}

by

Kathryn N Culliton, B. Eng

A thesis submitted to the Faculty of Graduate and Postdoctoral Affairs in partial fulfillment of the requirements for the degree of

Masters of Applied Science

in

Mechanical Engineering

Carleton University

Ottawa, Ontario

(C) 2020, Kathryn N Culliton 


\begin{abstract}
Solute transport has not been studied in articular cartilage subject to sliding loads, as occurs in vivo. Therefore, the goal of this thesis is to provide a comprehensive investigation of solute transport under sliding loads specifically investigating enhancement to mass transfer and spatiotemporal changes to diffusivity within the tissue. The findings are contextualized by supplemental investigations comparing the results to commonly studied loading modes such as uniaxial compression, elucidating the role of the superficial region as a barrier to load induced solute transport, and the accuracy of nutrient diffusion models (single vs multi-layer) for characterizing diffusivity. This study demonstrates that mass transfer is higher for sliding compared to uniaxial compression reaching 4.4-fold at $2 \mathrm{hrs}(\mathrm{p}=0.002)$ and this can exceed the passive capacity of the tissue. In addition, this work demonstrates a spatiotemporal dependence of diffusivity within the tissue under sliding loads.
\end{abstract}




\section{Preface}

Chapter 2 of this thesis "Sliding contact accelerates solute transport into the cartilage surface compared to axial loading" is currently under review with the Journal of Osteoarthritis and Cartilage (manuscript number OAC11038). The manuscript was submitted August $8^{\text {th }} 2020$ and is reproduced in whole for this chapter.

Chapter 3 of this thesis is currently in preparation for submission to the Journal of Osteoarthritis and Cartilage. 


\section{Acknowledgements}

I would first like to express my gratitude to my supervisor Andrew for his guidance and expertise. Your enthusiasm for research and problem-solving skills are unparalleled; both have been very helpful throughout this process. Lastly, thank you for giving me my first opportunity in research almost seven years ago; it has led to so many exciting career opportunities for which I am very grateful.

I would also like to thank Chloë van Oostende-Triplet and Skye McBride from the Cell Biology and Image Acquisition Core at the University of Ottawa, for their guidance developing the fluorescent image acquisition protocol. Trying to develop an imaging, histology, and mechanical protocol simultaneously was extremely challenging, but it was worth it when we could finally see the results. Thank you for the advice and conversation along the way. In addition, I would like to thank Sharlene Faulkes from the Louise Pelletier Histology Core Facility at the University of Ottawa for her assistance with the immunohistochemistry work. 


\section{Table of contents}

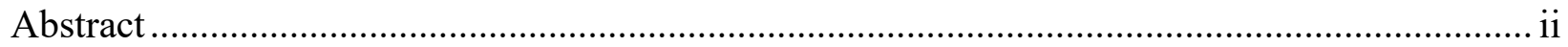

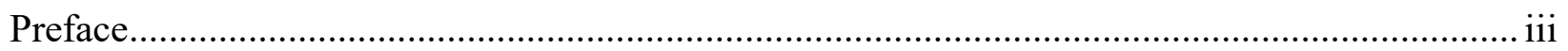

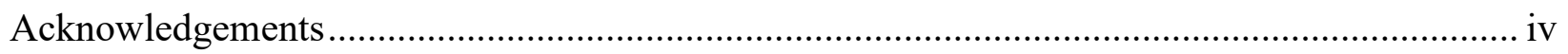

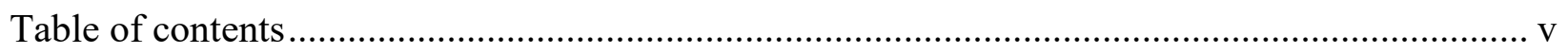

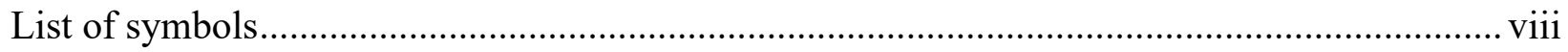

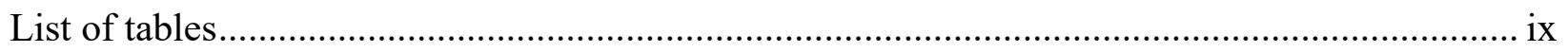

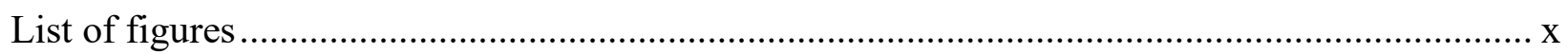

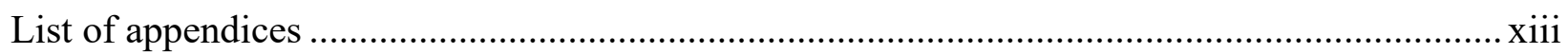



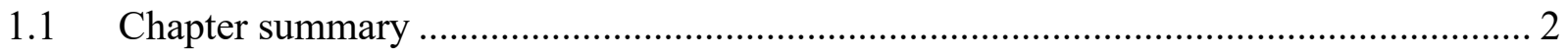



1.2.1 Structure and composition of articular cartilage ................................................ 4

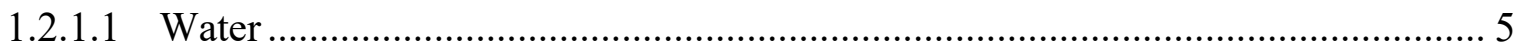

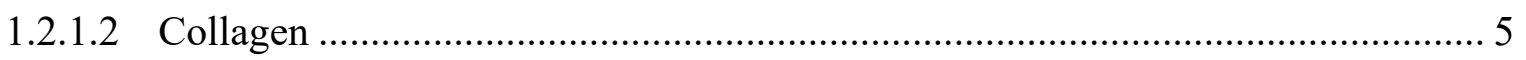

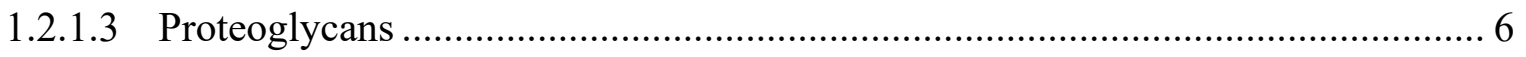

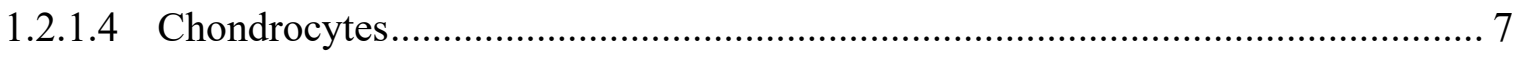

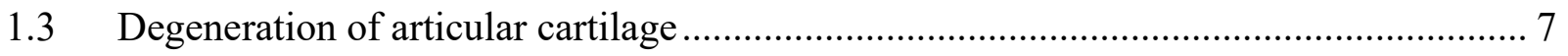

1.4 Solute transport in articular cartilage ................................................................ 9

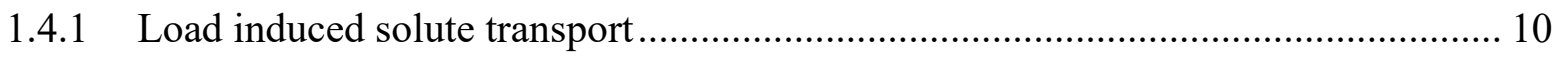

1.4.2 Parameters used to characterize solute transport in articular cartilage ................... 12

1.4.3 Modeling diffusivity in articular cartilage ....................................................... 13

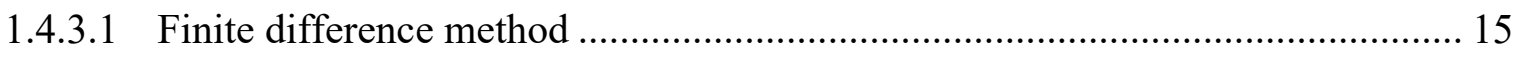

1.4.3.2 Transient multilayer one-dimensional diffusion equation .............................. 15



2 Sliding contact accelerates solute transport into the cartilage surface compared to axial

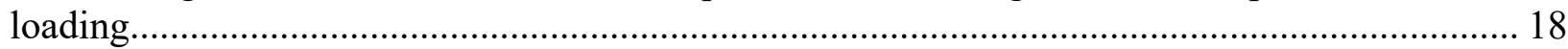

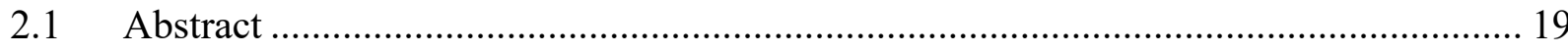

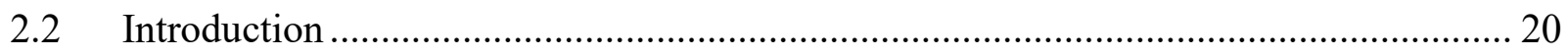

2.3 Materials and methods ................................................................................. 22

2.3.1 Tissue preparation ............................................................................................. 22

2.3.2 Tracer preparation ............................................................................................ 22

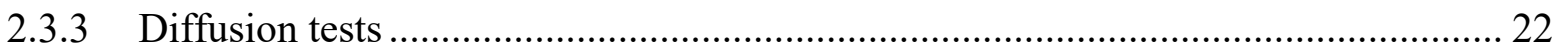


2.3.4 Immunohistochemical assessment of lubricin extraction ................................. 25

2.3.5 Fluorescent imaging and concentration profiles ............................................ 25

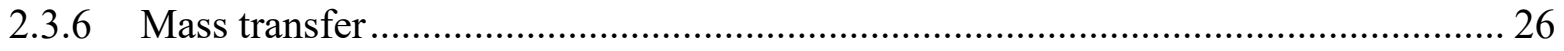

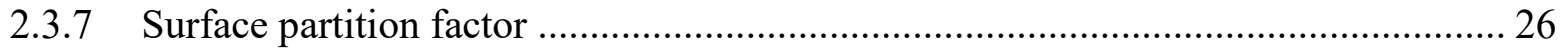

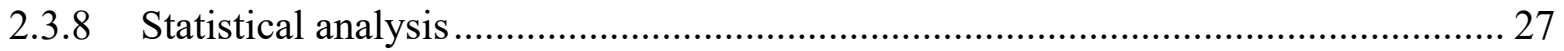

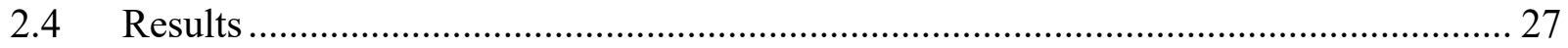

2.4.1 Observations between loaded conditions ......................................................... 27

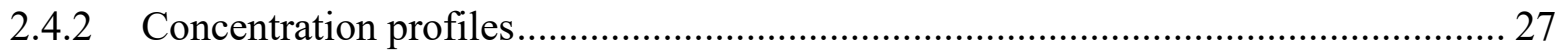

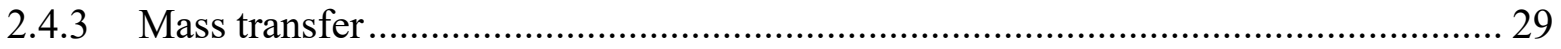

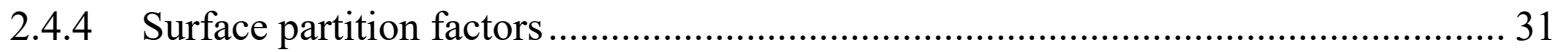

2.4.5 Immunohistochemical assessment of lubricin extraction .................................... 32

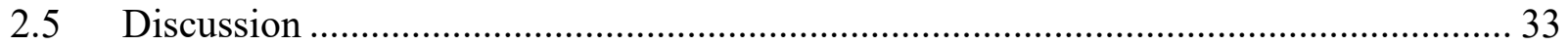

3 Multilayer nutrient diffusion modeling in articular cartilage subject to sliding and uniaxial

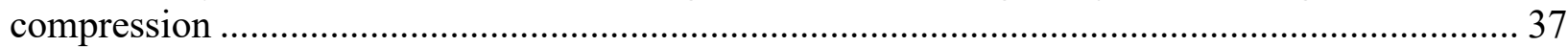





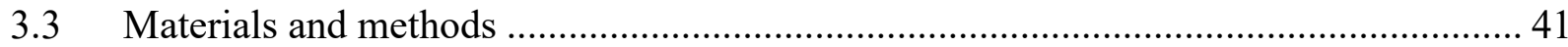

3.3.1 Specimen preparation............................................................................. 41

3.3.2 Mechanical protocol................................................................................ 41

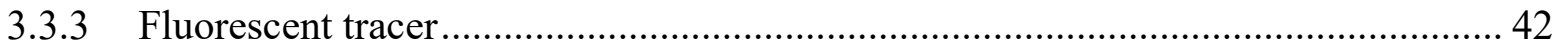

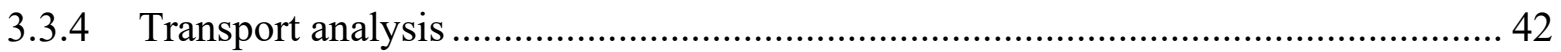

3.3.5 One-dimensional transient diffusion models ....................................................... 43





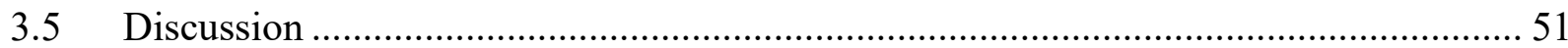

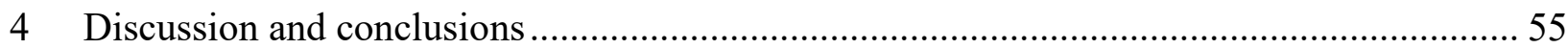

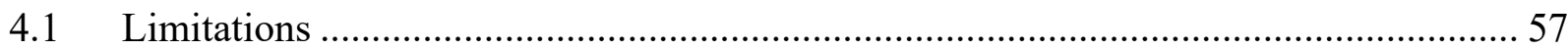

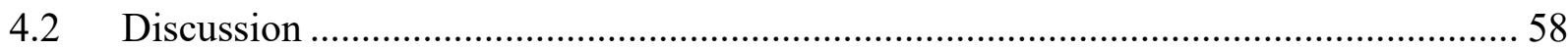

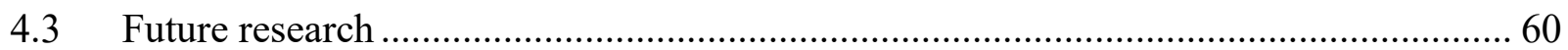

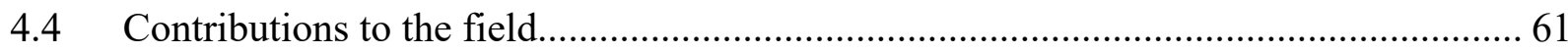




Nomenclature

\begin{tabular}{|c|c|}
\hline CT & Computed tomography \\
\hline ECM & Extracellular matrix \\
\hline FRAP & Fluorescent recovery after photobleaching \\
\hline GAG & Glycosaminoglycan \\
\hline HA & Hyaluronic acid \\
\hline MRI & Magnetic resonance imaging \\
\hline $\mathrm{OA}$ & Osteoarthritis \\
\hline PBS & Phosphate buffered saline \\
\hline PCM & Chondrocyte pericellular matrix \\
\hline PDE & Partial differential equation \\
\hline RA & Rheumatoid arthritis \\
\hline RMSE & Root mean squared error \\
\hline SHG & Second harmonic generation \\
\hline TBST & Tris-buffered saline \\
\hline TJR & Total joint replacement \\
\hline
\end{tabular}




\section{List of symbols}

$\begin{array}{ll}D & \text { Diffusivity }\left(\mu \mathrm{m}^{2} / \mathrm{s}\right) \\ D_{\text {eff }} & \text { Effective diffusivity }\left(\mu \mathrm{m}^{2} / \mathrm{s}\right) \\ C & \text { Concentration }(\mathrm{mg} / \mathrm{ml}) \\ x & \text { spatial dimension }(\mu \mathrm{m}) \\ v & \text { fluid velocity } \\ t & \text { time (s) } \\ J & \left.\text { Flux (mols/cm }{ }^{2} \mathrm{~s}\right) \\ K & \text { surface partition coefficient (unitless) } \\ C\left(l_{0}, t\right) & \text { Surface concentration (mg/ml) } \\ C_{0} & \text { Bath concentration (mg/ml) } \\ n & \text { nodes }\end{array}$




\section{List of tables}

Table 1-1: Studies that have investigated the effect of cyclic loading on solute transport in

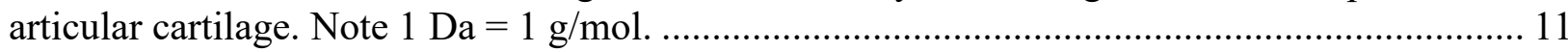




\section{List of figures}

Figure 1-1: Articular cartilage contacts of the knee joint during flexion. In full extension, there is central contact between the femoral condyles and the tibial plateau (A), when flexion is initiated the femoral condyles roll posteriorly (B) until the anterior cruciate ligament is engaged in tension at which point the femur is prevented from rolling further back and slides anteriorly (C).......... 4

Figure 1-2: Bright field cross-sectional image of articular cartilage from porcine patellar femoral grove. Image was obtained using an epifluorescence microscope under 5X magnification. Diagram (right) shows zonal organization of collagen fibers.

Figure 2-1: Experimental setup for dynamically loaded tests. Both conditions were maintained at a cycle frequency of $0.6 \mathrm{~Hz}$ for $0.5,1$, and $2 \mathrm{hr}$ durations. The radial edge of the tissue was sealed to restrict solute transport through the articular surface only. For samples subject to sliding, sections were obtained perpendicular to sliding direction. $\mathrm{SB}=$ subchondral bone, $\mathrm{AC}=$ articular surface

Figure 2-2: Fluorescent cross-sectional image of porcine articular cartilage following $2 \mathrm{hrs}$ of sliding with a migrating contact area. Image was obtained using an epifluorescence microscope under $5 \mathrm{X}$ magnification with a filter for fluorescein. Tracer accumulation in the pericellular matrix can be seen as bright spots around chondrocyte lacunae (arrow); concentration profiles are spatially averaged at each depth location and include these features.

Figure 2-3: Mean concentration profiles along the normalized length for (A) surface removed (B) intact. Shaded region shows $95 \%$ confidence interval of the mean. For the intact condition, samples sizes were 4, 7, and 6 for $12 \mathrm{hr}, 24 \mathrm{hr}$, and $72 \mathrm{hr}$ timepoints respectively. Samples sizes were 3, 6, and 3 for $12 \mathrm{hr}, 48 \mathrm{hr}$, and $72 \mathrm{hr}$ timepoints respectively for samples without a superficial region.

Figure 2-4: Mean concentration profiles along the normalized length for cyclic sliding (A) and cyclic compression (B). Shaded region shows $+95 \%$ confidence interval of the mean. All timepoints had 6 specimens per group except for sliding at $1 \mathrm{hr}(\mathrm{n}=5)$.

Figure 2-5: Mean mass transfer for all experimental groups and timepoints. Error bar shows $+95 \%$ confidence interval of the mean. Result of significant Mann Whitney U tests are displayed on plots.

Figure 2-6: Mean rate of mass transfer for all experimental groups and timepoints. Error bar shows $+95 \%$ confidence interval of the mean. Result of significant Mann Whitney $U$ tests are displayed on plots.

Figure 2-7: Mean surface partition factor for all experimental groups and timepoints. Error bar shows $+95 \%$ confidence interval of the mean. Result of significant Mann Whitney U tests are displayed on plots.

Figure 3-1: Cross sectional schematic of experimental set up. Osteochondral plugs were sealed around the radial edge to prevent solute uptake from this direction. Fluorescent tracer could only enter the tissue through the bearing surface. Arrows indicate direction of motion and dynamic loads: sliding motion (left) and uniaxial compressive force (right). 
Figure 3-2: Schematic showing experimental conditions for multilayer diffusion model. $m$ indicates the layer number. Single layer is the same except the tissue is not subdivided into multiple layers as indicated by the dashed lines.

Figure 3-3: Mean concentration profiles following 0.5, 1, and $2 \mathrm{hrs}$ of biaxial sliding, shaded region represents $95 \%$ confidence interval of the mean. A) shows fit obtained using a leastsquares approximation to the 1D transient diffusion model using a single layer model. B) shows fits obtained using a least-squares approximation to the 1D transient diffusion model using a multilayer model. To best visualize the difference between the multilayer and single layer models, the fits obtained for this figure were to the mean intensity profile at each time point. See Appendix B for results from individual specimens. 46

Figure 3-4: Mean concentration profiles for normalized depth (x/l) following $0.5,1$, and $2 \mathrm{hrs}$ of cyclic uniaxial compression, shaded region represents $95 \%$ confidence interval of the mean. A) shows fit obtained using a least-squares approximation to the 1D transient diffusion model using a single layer model. B) shows fits obtained using a least-squares approximation to the $1 \mathrm{D}$ transient diffusion model using the multilayer model. To best visualize the difference between the multilayer and single layer models, the fits obtained for this figure were to the mean intensity profile at each time point.

Figure 3-5: Mean RMSE of experimental groups comparing fit obtained from multilayer and single layer models. Bar represents $95 \%$ confidence interval of the mean.

Figure 3-6: Diffusivity as a function of depth for samples subject to sliding (A) and cyclic compression (B). Diffusivity was determined using a least-squares approximation to the 1D transient multilayer diffusion model. Layers 18-20 are not shown due to the numerical errors associated with fitting a diffusivity in a plateau region of a concentration profile.

Figure 3-7: Diffusivity for experimental groups determined using a least-squares best fit to the 1D transient diffusion model. A single diffusivity was fit through the depth of the tissue......... 50

Figure A-1: Calibration curve generated for converting fluorescent intensity to concentration. . 71

Figure B-1: Mean concentration profiles for specimens subject to the lubricin extraction protocol. Shaded region shows $+95 \%$ confidence interval of the mean. Samples sizes were 7, 6, and 6 for $6 \mathrm{hr}, 12 \mathrm{hr}$, and $24 \mathrm{hr}$ timepoints respectively.

Figure B-2: Mean mass transfer, rate of mass transfer and surface partition factor for specimens subject to lubricin extraction protocol. Error bar shows $+95 \%$ confidence interval of the mean. 74

Figure C-1: Sensitivity analysis for determining the number of subdivisions within the multilayer model. 20 layers were chosen as a compromise between accuracy and computational time. Results shown for 4 randomly selected samples.......................................................... 76

Figure D-1: Single layer model fits to intensity profiles for samples subject to sliding. Dashed line indicates model fit, solid line represents experimental data. Experimental time points $0.5 \mathrm{hr}$ (top), $1 \mathrm{hr}$ (middle), and $2 \mathrm{hr}$ (bottom).

Figure D-2: Multilayer model fits to intensity profiles for samples subject to sliding. Dashed line indicates model fit, solid line represents experimental data. Experimental time points $0.5 \mathrm{hr}$ (top),

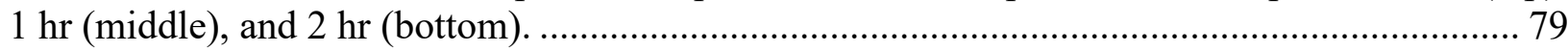


Figure D-3: Single layer model fits to intensity profiles for samples subject to uniaxial compression. Dashed line indicates model fit, solid line represents experimental data. Experimental time points $0.5 \mathrm{hr}$ (top), $1 \mathrm{hr}$ (middle), and $2 \mathrm{hr}$ (bottom).

Figure D-4: Multilayer model fits to intensity profiles for samples subject to uniaxial compression. Dashed line indicates model fit, solid line represents experimental data. Experimental time points $0.5 \mathrm{hr}$ (top), $1 \mathrm{hr}$ (middle), and $2 \mathrm{hr}$ (bottom). 


\section{List of appendices}

Appendix A: Conversion from fluorescent intensity to concentration .................................... 70

Appendix B: Results of solute transport following lubricin removal ....................................... 72

Appendix C: Multilayer model sensitivity analysis to determine number of layers................... 75

Appendix D: Single layer and multilayer model fits to all specimens ..................................... 77 


\section{Introduction}




\subsection{Chapter summary}

Articular cartilage makes up the bearing surface of synovial joints and provides a low friction, high load bearing interface between adjacent bones. Its structure and composition provide the unique properties that, in the absence of disease, allow the tissue to undergo minimal deterioration in mechanical properties with normal aging. The most common disease affecting articular cartilage is arthritis which is characterised by degeneration of the bearing surface resulting in pain and loss of function. Currently, degenerative diseases of articular cartilage such as arthritis are irreversible with limited non-surgical treatment options. One of the primary challenges to preventing and treating arthritis is the lack of vascularization within the tissue. The avascular nature means that all nutrients and therapeutics required to support cell activity can only reach the embedded chondrocytes via diffusion. This limits the capacity of the tissue to selfrepair and creates challenges delivering therapeutics. Therefore, both understanding the etiology of the disease, and developing therapeutics to treat arthritis, rely on a comprehensive understanding of solute transport pathways within the tissue. This has been an extensive focus of cartilage research for many decades. Several factors have been identified that influence transport kinetics including solute shape, charge and size, cartilage composition, and load application. It has been established that movement, specifically cyclic compression, recovers interstitial fluid lost during static loading and enhances the uptake of solutes into the cartilage matrix. However, pure cyclic compression is largely absent in normal joint motion and therefore studies focusing on this loading mode do not provide a complete understanding of solute transport that occurs during normal daily function. Only recently have the contributions of sliding, a predominate component of joint articulation been considered. Currently literature lacks an understanding of how sliding influences mass transfer, surface partitioning and diffusivity within the tissue. This 
is an important area of research as it will have significant implications on biochemical signaling, nutrient transport, drug delivery, and engineered cartilage.

\subsection{Articular cartilage of synovial joints}

Articular cartilage is a highly specialized form of hyaline cartilage found at the end of bones in synovial joints. This tissue provides a low friction bearing surface for joint articulation and facilitates load transfer between adjacent bones. In human hip and knee this tissue ranges between 1.4-2.6 mm thick for healthy tissue [1]. The joint is encased by a fibrous capsule that is continuous with the synovial membrane. This membrane secretes synovia, a viscous, nonNewtonian fluid, that provides lubrication and nutrients to the encapsulated tissue. Under load, the boundary lubricants (Figure 1-1) facilitate low friction joint motion and alter the shear kinematics of sliding [2]. The joint is stabilized by ligaments which prevent abnormal joint motions and facilitate movement. Normal joint motion results in two primary types of cartilage contact, rolling and sliding (Figure 1-1). During gait, both contacts are accompanied by a varying axial load due to changes in weight bearing through the gait cycle. 
A

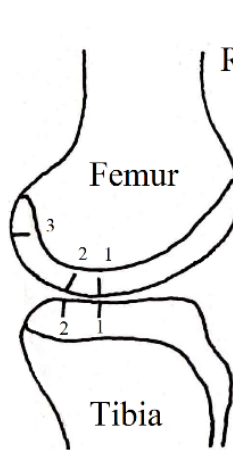

B

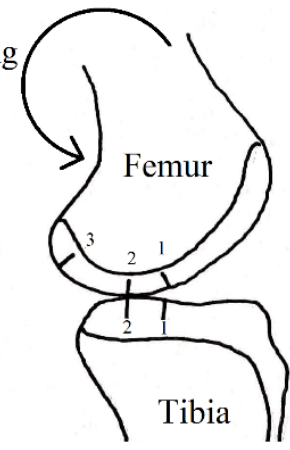

C

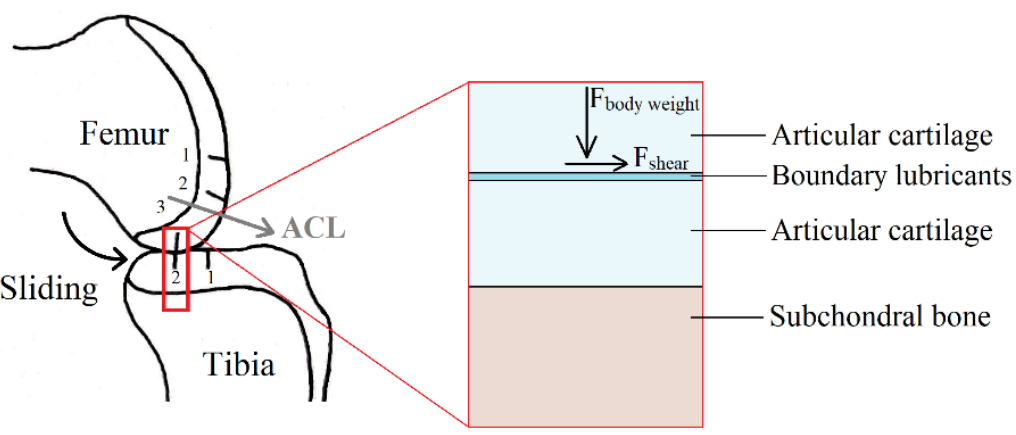

Figure 1-1: Articular cartilage contacts of the knee joint during flexion. In full extension, there is central contact between the femoral condyles and the tibial plateau (A), when flexion is initiated the femoral condyles roll posteriorly (B) until the anterior cruciate ligament is engaged in tension at which point the femur is prevented from rolling further back and slides anteriorly (C).

\subsubsection{Structure and composition of articular cartilage}

The composition and spatial arrangement of cartilage provides the necessary properties to withstand high tensile and compressive loads as well as resistance to wear. In the absence of disease, cartilage can endure a lifetime of load cycles with minimal losses to function [3], [4]. The tissue is composed of a dense extracellular matrix (ECM) with a sparse distribution of specialized cells known as chondrocytes throughout. Based on collagen fibril organization, the tissue is divided into three primary regions: superficial ( $\sim 15 \%$ of the tissue depth), middle transitional region ( $\sim 60 \%$ of the tissue depth), and deep zone (remainder of the tissue depth) which is anchored to the subchondral bone by a calcified region.

Articular cartilage is often described as a biphasic material containing a solid matrix and fluid phase [5]. The solid matrix is characterized by the densely packed collagen-proteoglycan network, and water content constitutes the fluid phase. Under load, interstitial fluid pressure increases due to the low permeability of the tissue that prevents rapid exudation of fluid. The 
high osmotic pressure in the fluid matrix provides resistance to compressive stress [6]; this is reinforced by the collagen fibril network which is responsible for providing resistance to shear and tensile forces.

\subsubsection{Water}

Water accounts for approximately $80 \%$ of articular cartilage by weight[7]-[9]. Dissolved within the water are inorganic ions such as sodium, calcium, chloride and potassium needed to maintain osmolarity and chondrocyte function [10]. Approximately $30 \%$ of the water exists in the intrafibrillar space [11], a very small percentage exists in the intracellular space (due to the sparsity of chondrocytes), and the remainder constitutes the pore space of the tissue. The pressurization of water within the tissue contributes to its ability to withstand high loads. During joint loading and concomitant fluid pressurization, interfibrillar water is cyclically moved through the ECM via pressure gradients allowing nutrients to be delivered to the chondrocytes. Water concentration decreases depth-wise within the tissue due to increasing proteoglycan content, starting at approximately $80 \%$ in superficial regions decreasing to $65 \%$ in the deep zone [9], [12].

\subsubsection{Collagen}

The extracellular matrix is primarily made up of collagen, accounting for $60 \%$ of the dry weight of the tissue [7]; most of which is type II collagen (90-95\%). The collagen network has a zonal arrangement that varies as a function of tissue depth. At the surface, collagen fibers are parallel to the bearing surface; this arrangement exists for approximately $15 \%$ of the tissue depth [13]. The primary role of this region is to provide the tensile properties needed to resist shear forces during articulation and resist lateral expansion under compressive loads, thereby increasing fluid 
load support. The transitional zone makes up $60 \%$ of the tissue depth, in this region the collagen fibers are oriented randomly. In the remaining portion of the tissue, the deep zone, the fibers are oriented perpendicular to the bearing surface and are anchored into the subchondral bone by a calcified region [13] (Figure 1-2).

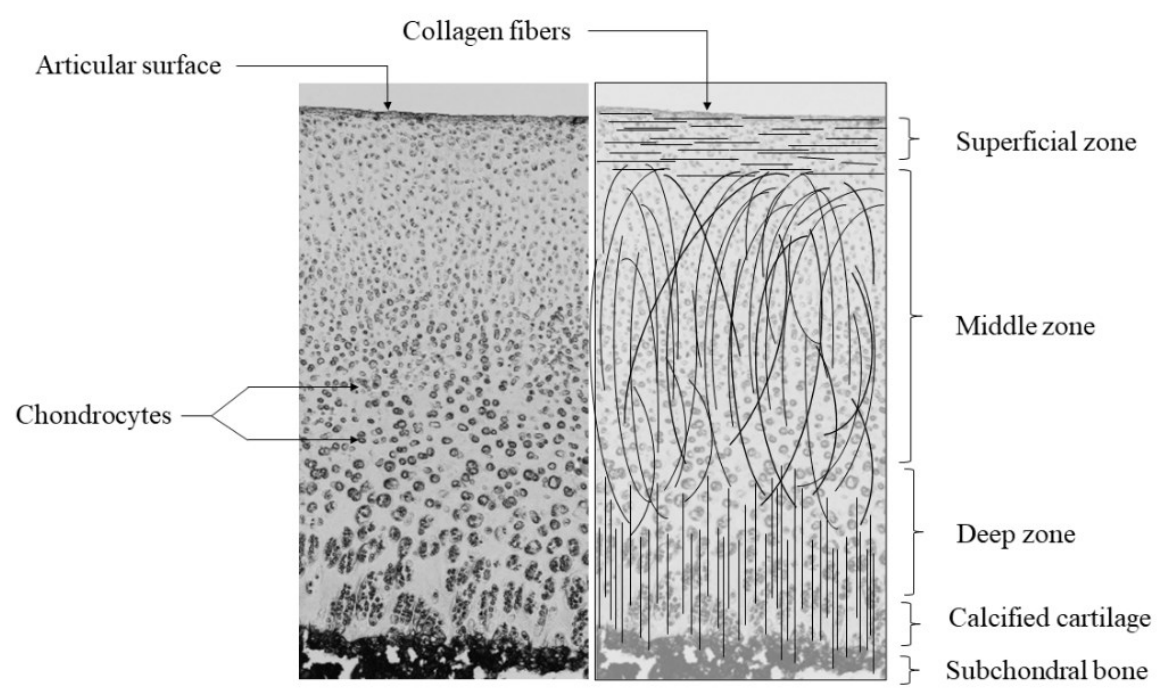

Figure 1-2: Bright field cross-sectional image of articular cartilage from porcine patellar femoral grove. Image was obtained using an epifluorescence microscope under $5 \mathrm{X}$ magnification. Diagram (right) shows zonal organization of collagen fibers.

\subsubsection{Proteoglycans}

Proteoglycans are glycosylated macromolecules that contain a central protein with one or more glycosaminoglycan (GAG) chains. These macromolecules create electrostatic forces within the tissue and are fundamental to the tissue's load carrying capacity [6], [14]. Two primary types of interactions exist: attraction between the negatively charged GAG chains and bipolar water molecules, and electrostatic repulsion between neighbouring GAG chains. The former results in the high-water content with both contributing to the low hydraulic permeability of the tissue. Proteoglycan content is directly proportional to the fixed charge density, which increases with depth from the articular surface [15]. 
In addition, proteoglycans also exist on the surface of articular cartilage and play a large role in joint lubrication. These specialized surface proteoglycans are known as lubricin or proteoglycan 4. Lubricin interacts with hyaluronic acid (HA) and phosphatidylcholine lipid complexes to create an extremely low coefficient of friction when articulated against one another [16]. As a result, lubricin deficiencies alter joint lubrication which can have cascading effects on fluid exchange with the surrounding synovia and ultimately solute transport.

\subsubsection{Chondrocytes}

Chondrocytes are highly specialized cells found within articular cartilage that are responsible for the development, maintenance, and turnover of the ECM within their vicinity. These cells originate from mesenchymal stem cells and are sparsely distributed throughout the volume of the tissue. The sparsity and inability of the cells to migrate within the tissue results in minimal contact between neighbouring chondrocytes. Chondrocytes are heavily influenced by their chemical and mechanical environment and it has been established that they are responsive to stimuli such as growth factors [17], mechanical loads [18], [19], and hydrostatic pressures [20], [21]. With no direct blood supply within cartilage, chondrocytes rely on diffusion for the supply of nutrients, biochemical signals, and waste removal.

\subsection{Degeneration of articular cartilage}

Despite the impressive structural properties and complex composition, mature cartilage has a very limited capacity to self repair. This is often attributed to the lack of vascularization and lymphatics, and the inability of chondrocytes to migrate to areas in need of repair. Degeneration of articular cartilage primarily occurs in the form of arthritis, the most common form being osteoarthritis (OA) which is estimated to affect approximately $10 \%$ of Canadian adults [22]. 
Current treatment strategies for the disease are limited, with most nonoperative management techniques having poor clinical outcome. Ultimately, most patients will require total joint replacement (TJR) to completely alleviate pain and restore joint function. One of the primary barriers to providing effective therapeutic strategies against $\mathrm{OA}$ is the inability to detect $\mathrm{OA}$ prior to significant degeneration. Currently OA is diagnosed by joint space narrowing on projection radiographs; radiographic techniques are limited to identifying gross changes in the osseous anatomy that occur at a late and irreversible stage of the disease.

Early in the degenerative process, prior to symptom onset, the composition and structure of the tissue is altered, progressing to significant changes in the mechanical properties [23]-[25]. Histological techniques have been able to identify changes to the cartilage early in the OA process, including a decrease in proteoglycan content [26] and change in the collagen architecture [27], [28]. The highly organized spatial arrangement of the collagen fibers is lost during the degenerative process due to cleavage of the fibrils. However, histological techniques are limited to ex vivo experiments and do not translate to a clinical diagnosis.

There has been some progress developing strategies to detect OA prior to significant loss of function, namely magnetic resonance imaging (MRI) based biomarkers for proteoglycan and collagen content. Establishing MRI biomarkers for OA is advantageous as they are non-invasive and are an earlier indicator of degeneration than traditional radiographic techniques. MRI is routinely used to assess joint anatomy as well as degeneration of the cartilage in vivo using several techniques including T2 and T1rho weighted imaging sequences. Both T2 and T1rho values are elevated in patients with OA compared to healthy controls and have been correlated with increased severity in radiographic and MRI grading for OA [29]. Unfortunately, the 
technology is limited by low specificity. As contrast agents are developed to improve early diagnosis of OA, understanding pathways of solute transport within the tissue is a vital part to their success.

\subsection{Solute transport in articular cartilage}

As an avascular tissue, articular cartilage relies on diffusion pathways to provide nutrients and molecules necessary for cellular activity. These pathways are key to maintaining and improving the health of articular cartilage and ultimately synovial joints. There are two primary components that affect solute transport in articular cartilage: the properties of the diffusing solute and the composition of the tissue. It is well established that solute size (molecular weight and hydrodynamic radius), shape (linear vs spherical), and charge (anionic, cationic, neutral) all influence diffusion [30]-[37].

Articular cartilage is a heterogeneous medium with spatial composition varying as a function of tissue depth. Local and bulk changes to the tissue composition have been attributed to altered transport kinetics [33], [38]-[40]. In healthy tissue, diffusion coefficients vary significantly with cartilage zone; interestingly, the variation is not consistent across different sized solutes [38]. During the degenerative process, significant changes occur to the cartilage matrix resulting in concomitant changes to solute transport. Decreased proteoglycan content seen in OA has been associated with increased diffusivity, this is thought to be a result of the increase in effective pore size [31]. Other changes, localized to the superficial region, have also been associated with altered diffusivity. Disruption to the collagen architecture has resulted in a significant increase in diffusivity, indicating that collagen at the surface of cartilage acts as a barrier to solute transport 
[40]. In addition, other disruptions to the articular surface such as small fissures induced by mechanical injury have lead to enhanced transport across the articular surface [32].

\subsubsection{Load induced solute transport}

Solute transport is highly dependent on the mechanical behavior of articular cartilage under load and concomitant fluid recovery. During axial compression, deformation causes pressurization of the fluid within the tissue. This occurs due to the low hydraulic permeability providing resistance to fluid flow within the ECM. Over time the collapsing pore space will exude fluid into the joint space. This fluid is recovered when the applied load is removed. The exchanging of fluid between the tissue and synovia provides nutrients and other molecules needed for cellular activity. Other components of joint motion such as sliding, also play a role in fluid recovery, although the mechanisms by which this occurs are not as well understood. Tribological rehydration is the fundamental mechanism behind interstitial fluid recovery during sliding in the absence of varying load amplitude and contact migration [41], [42]. Fluid can be retained and recovered by this process due to competition between elasto-hydrodynamic pressure driven inflow and interstitial exudation. Fluid recovery that occurs via sliding with a migrating contact area is likely a combination of the two mechanisms. Interstitial fluid recovery during dynamic loading (sliding or uniaxial compression) is the fundamental principle behind physical activity promoting and maintaining joint health [43].

Most studies that have looked at the effect of mechanical loading on solute transport in articular cartilage have largely focused on uniaxial cyclic or static compression [33], [44]-[47] (Table 1-1). DiDomenico et al. have shown that loading significantly increases diffusivity throughout the tissue, with the maximum increases occurring around $257 \mu \mathrm{m}$ from the articular surface [33]. 
Though it is a predominate component of joint motion, only recently have the contributions of sliding, in relation to solute transport, been considered. Burris et al. have studied the process of tribological rehydration and demonstrated it is the primary driver of solute transport, cartilage thickening, and friction reduction under sliding contact [41], [42], [48]. Their work largely focuses on describing the occurring phenomenon, as opposed to providing a quantitative comparison to other loading modes such as cyclic compression. Their method isolates for pure sliding due to the absence of a varying load amplitude. Furthermore, the contact location on the cartilage remains static as a glass slide moves over the cartilage surface. Therefore the in vivo translation of their work is limited. Lacking a quantitative comparison, it remains unclear what component of joint motion, sliding or cyclic uniaxial compression, is a larger driver of solute transport.

Table 1-1: Studies that have investigated the effect of cyclic loading on solute transport in articular cartilage. Note $1 \mathrm{Da}=1 \mathrm{~g} / \mathrm{mol}$.

\begin{tabular}{|c|c|c|c|}
\hline Study & Loading mode(s) & $\begin{array}{l}\text { Solute(s) used } \\
\text { (molecule size, } \\
\text { charge) }\end{array}$ & Findings \\
\hline [41] & $\begin{array}{l}\text { - Sliding with a static } \\
\text { contact area (1- } \\
80 \mathrm{~mm} / \mathrm{s}) \\
\text { - Static load }(5-10 \% \\
\text { strain) }\end{array}$ & $\begin{array}{l}\text { AlexaFluor } 633 \\
\text { (1.2 kDa, neutral) }\end{array}$ & $\begin{array}{l}\text { Following static load (exudation), } \\
\text { sliding at speeds of } 60 \mathrm{~mm} / \mathrm{s} \\
\text { increased solute uptake into buried } \\
\text { contacts. This was orders of } \\
\text { magnitude greater than passive } \\
\text { diffusion. }\end{array}$ \\
\hline [47] & $\begin{array}{ll}\text { - } & \text { Unloaded } \\
\text { - } & \text { Static load }(10 \% \\
\text { strain }) \\
\text { - } \\
\text { Cyclic uniaxial } \\
\text { compression }(0-10 \% \\
\text { strain). }\end{array}$ & $\begin{array}{l}\text { Iohexol } \\
\text { (821 Da, neutral) }\end{array}$ & $\begin{array}{l}\text { Solute uptake is influenced by } \\
\text { loading mode in arthritic samples. } \\
\text { For early and mid stage OA, cyclic } \\
\text { uniaxial compression accelerated } \\
\text { solute transport compared to static } \\
\text { loading. In late stage OA cyclic } \\
\text { loading had no affect on solute } \\
\text { uptake. }\end{array}$ \\
\hline
\end{tabular}




\begin{tabular}{|c|c|c|c|}
\hline [45] & $\begin{array}{l}\text { - Cyclic uniaxial } \\
\text { compression }(0.25-5 \% \\
\text { strain, at } 0.25-2.6 \mathrm{~Hz}) \\
\text { - Static load }(15 \% \\
\text { strain) }\end{array}$ & $\begin{array}{l}\text { Alexa Fluor } 633 \\
\text { (150 kDa, neutral) }\end{array}$ & $\begin{array}{l}\text { In this study solute transport was } \\
\text { studied in the radial direction } \\
\text { (parallel to the articular surface). } \\
\text { Load was applied axially. Cyclic } \\
\text { uniaxial compression enhanced solute } \\
\text { transport 2-3 fold compared to } \\
\text { passive diffusion under static load. } \\
5 \% \text { strain at } 1 \mathrm{~Hz} \text { had induced highest } \\
\text { levels of solute transport. }\end{array}$ \\
\hline [46] & $\begin{array}{l}\text { - Cyclic uniaxial } \\
\text { compression (applied } \\
\text { radially, } 1.25-5.0 \% \\
\text { strain at } 1 \mathrm{~Hz}) \\
\text { - Static load }(15 \% \\
\text { strain) }\end{array}$ & $\begin{array}{l}\text { Alexa Fluor } 633 \\
\text { variants } \\
(150 \mathrm{kDa} \text {, negative } \\
4.7-5.9 \mathrm{IP})\end{array}$ & $\begin{array}{l}\text { Solute transport was increased when } \\
\text { samples were subject to cyclic } \\
\text { compression. Isoelectric point only } \\
\text { had a significant effect on diffusivity } \\
200-275 \mu \mathrm{m} \text { from the articular } \\
\text { surface. }\end{array}$ \\
\hline [44] & $\begin{array}{l}\text { - Cyclic uniaxial } \\
\text { compression }(2.8 \mathrm{MPa} \\
\text { at } 1 \mathrm{~Hz}) \\
\text { - Unloaded }\end{array}$ & $\begin{array}{l}\text { Urea }(60 \mathrm{Da}) \\
\mathrm{NaI}(125 \mathrm{Da}) \\
\text { Serum albumin (66 } \\
\text { kDa) }\end{array}$ & $\begin{array}{l}\text { Cyclic compression had no effect on } \\
\text { the absorption or desorption of small } \\
\text { solutes. The rate of desorption of } \\
\text { large solutes (serum albumin) was } \\
\text { increased } 30-100 \% \text {. }\end{array}$ \\
\hline [49] & $\begin{array}{l}\text { - Unloaded } \\
\text { - Cyclic uniaxial } \\
\text { compression }(5-50 \% \\
\text { strain at, 0.0006-0.1 } \\
\text { Hz) }\end{array}$ & $\begin{array}{l}\text { Fluorescent } \\
\text { glucose analogue } \\
(342 \mathrm{Da})\end{array}$ & $\begin{array}{l}\text { Maximum increase in solute } \\
\text { desorption was achieved with } 10- \\
20 \% \text { compression at } 0.1 \mathrm{~Hz} \text {. }\end{array}$ \\
\hline
\end{tabular}

\subsubsection{Parameters used to characterize solute transport in articular cartilage}

Several parameters are used to characterize solute transport in articular cartilage namely, mass transfer, surface partition, and diffusivity. Mass transfer is representative of the total amount of a solute within the tissue and would be analogous to total nutrient or exogenous biochemical supply in vivo. The metric is easy to assess provided intensity profiles generated by the methods used (e.g. MRI, computed tomography (CT), fluorescent microscopy) can be converted to a concentration or a quantitative amount. Total mass transfer will increase until samples have 
reached equilibrium. While approaching equilibrium, rate of mass transfer decreases with increasing experimental duration. Another useful metric for characterizing solute transport is the surface partition factor. This characterizes the concentration interface between the tissue and the solute bath and represents the permeability of the superficial region. In most cases, there will be a jump boundary condition at this interface. This factor was first described by Torzilli et al. and is calculated as a ratio between the concentration at the exposed surface and the bath [50]. This differs from the more commonly used, partition factor, which is the ratio of solute remaining in the tissue to solute in the surrounding fluid bath following desorption. By far, the most common metric used to assess solute transport in articular cartilage is diffusivity which represents the ability of a solute to diffuse through a given medium. While it is useful for capturing transport within the medium, alone this metric fails to capture solute transport for the whole system.

\subsubsection{Modeling diffusivity in articular cartilage}

Diffusion is the movement of solutes along a concentration gradient. This process occurs due to Brownian motion, which causes the particles to move randomly, eventually dispersing themselves evenly within the medium. In steady-state conditions, this is modeled by Fick's first law of diffusion (Eq. 1-1):

$$
J=-D \frac{\partial C}{\partial x}
$$

where $J$ is the diffusion flux (amount of substance/area/time), $C$ is the concentration of the diffusing substance per unit volume, $t$ is time, $x$ is the spatial dimension of the material in which diffusion is occurring, and $D$ is diffusivity (unit area/s). In articular cartilage, diffusion is a nonsteady state process which causes the concentration of the diffusing solute to change with respect 
to time within the tissue. Therefore, in articular cartilage, diffusion is characterized by Fick's second law for non-steady state diffusion (Eq. 1-2). For one dimensional transport this can be expressed:

$$
\frac{\partial C}{\partial t}=D \frac{\partial^{2} C}{\partial x^{2}}-v \frac{\partial C}{\partial x}
$$

where $C$ is the concentration of the diffusing substance per unit volume, $t$ is time, $x$ is the spatial dimension of the material in which diffusion is occurring, $D$ is diffusivity (unit area/s), and $v \frac{\partial C}{\partial x}$ is the contribution from advection under fluid velocity, $v$.

The diffusion coefficient, $D$, is typically used in cartilage research to describe and compare the movement of various solutes through the tissue. There are several limitations modelling diffusion within articular cartilage according to Fick's second law (Eq. 1-2). First, diffusion within the tissue occurs in the fluid filled pore-space. Effective pore space depends on local composition and charge which vary through the depth of the tissue [51]. In addition, free water (non collagen bound water) changes with collagen content within the tissue. In loaded conditions, especially those that result in tissue consolidation (i.e., cyclic uniaxial compression, static compression), the pore space decreases with duration of loading. Interstitial fluid is exuded from the tissue as the pore space collapses resulting in a compositional change to the tissue and a further decrease in available space for solute transport. In general, loading at the surface causes tissue compression, and hence pore volume decrease, that varies through the depth. This compositional change would likely result in a time-dependent diffusivity. In addition, in loaded conditions there are contributions from both passive and advective transport. The contributions from advection are challenging to measure experimentally and therefore, non passive conditions produce an 
"effective diffusivity," $D_{e f f}$, which incorporates the effects of advection (Eq. 1-3). This term is typically used to compare with passive diffusivities in literature:

$$
\frac{\partial C}{\partial t}=D_{e f f} \frac{\partial^{2} C}{\partial x^{2}}
$$

\subsubsection{Finite difference method}

Several analytical and numerical solutions exist to solve the governing partial differential equation (PDE). Analytical solutions can be obtained for a number of initial and boundary conditions [52]. The finite difference methods are commonly used to numerically solve the PDE (Eq. 1-3) for any initial or boundary condition. A Crank-Nicolson scheme uses a forward difference method in time and second order central difference approximation in space. The discretization is as follows:

$$
\frac{C_{i}^{n+1}-C_{i}^{n}}{\Delta t}=\frac{D_{e f f}}{2(\Delta x)^{2}}\left(\left(C_{i+1}^{n+1}-2 C_{i}^{n+1}+C_{i-1}^{n+1}\right)+\left(C_{i+1}^{n}-2 C_{i}^{n}+C_{i-1}^{n}\right)\right)
$$

\subsubsection{Transient multilayer one-dimensional diffusion equation}

Articular cartilage is a heterogeneous medium that benefits from the multilayer transient onedimensional diffusion model developed by Carr and Turner [53]. This model has successfully been used to determine depth-wise diffusivity of articular cartilage [33], [40]. Briefly, their model uses a vertex centered finite volume method with a backward Euler discretization in time. The method creates a mesh over each layer containing $n$ nodes; for simplicity, equal spacing is assumed between nodes. This method can account for varying transport properties through the depth of cartilage due to varying composition and has been used previously. 
Prior to the multilayer model developed by Carr and Turner, diffusivity had only been studied in the three primary regions of the tissue, superficial, middle and deep [38]. These results were achieved using fluorescent recovery after photobleaching (FRAP). Ultimately, this technique does not permit the continuous assessment of diffusivity from surface to subchondral bone. When the multilayer technique was applied to articular cartilage by DiDomenico et al., they were able to assess graduated depth dependent diffusivity and locate peak diffusivities[33], [40], [46].

\subsection{Thesis goals}

Since solute transport has not been studied in cartilage with sliding loads, as occurs in vivo, the goal of this thesis is to compare the mass transfer of a small, nutrient-sized molecule, under sliding and uniaxial loads as well as under free diffusion. The effect of the surface layer on transport can be assessed by using surface treatments to remove lubricin and the superficial region. A second goal is to compare the accuracy of nutrient diffusion models (single vs multilayer) for representing load-induced diffusion. Specifically, the goals of this thesis are:

1. To quantitatively compare solute uptake driven by sliding with a migrating contact area to cyclic uniaxial compression.

2. To evaluate the role of the superficial region on passive diffusion and compare to the results from loaded tests.

3. To compare the accuracy of nutrient diffusion models (single vs multi-layer) for representing load induced diffusion.

4. To evaluate depth dependent diffusivity of articular cartilage following sliding and uniaxial compression.

Research goals 1 and 2 are covered in Chapter 2 of this thesis. We hypothesize that: 
1. Solute uptake would be highest under sliding with a migrating contact area, followed by uniaxial compression.

2. The superficial region acts as a barrier to diffusion and sliding is overcomes not only the low permeability, but also the passive capacity of the tissue.

Research goals 3 and 4 are covered in Chapter 3 of this thesis. We hypothesize that:

3. Due to the heterogeneity of the tissue, a multilayer diffusion model will best describe load induced diffusion for both loading modes.

4. Sliding and uniaxial compression will result in varying diffusivity with tissue depth, and this will differ between loading modes. 


\section{Sliding contact accelerates solute transport into the cartilage surface compared to axial loading}

Under Review: Journal of Osteoarthritis and Cartilage 


\subsection{Abstract}

Objective: The objectives of this study were: first, to compare solute uptake driven by sliding to cyclic uniaxial compression. And secondly, to evaluate the role of the superficial region on passive diffusion to determine if mechanical action is merely overcoming the low permeability of the superficial region or exceeding equilibrium capacity of the tissue.

Design: Tests were performed on osteochondral plugs under two types of conditions: cyclic loading (sliding vs axial compression) and unloaded passive diffusion (intact vs superficial zone removed). The articular surfaces were exposed to a fluorescent bath and uptake was quantified from the surface to the subchondral bone using fluorescent microscopy. Primary outcome measures were total mass transfer, mass transfer rate, and surface partition factor.

Results: Mass transfer was 2.1-fold higher at $0.5 \mathrm{hr}$ for sliding compared to uniaxial compression $(\mathrm{p}=0.004)$. This increased to 4.4 -fold at $2 \mathrm{hrs}(\mathrm{p}=0.002)$. Solute transport for both loading conditions at $2 \mathrm{hrs}$ had reached or exceeded intact passive diffusion at $12 \mathrm{hrs}$. Total mass transfer and rate of mass transfer were higher in samples without the superficial region compared to intact samples at equilibrium. Rate of mass transfer was not declining for samples subject to sliding indicating solute uptake induced by sliding would exceed passive tissue capacity.

Conclusions: These results are the first to quantify solute uptake between two components of joint articulation. The study demonstrates that sliding is a larger driver of solute transport compared to cyclic uniaxial compression. This has implications for cell nutrition, tissue engineering and biochemical signaling.

Keywords: Cartilage, solute transport; sliding contact; axial loading 


\subsection{Introduction}

Due to the avascular nature of articular cartilage, the tissue relies on diffusion pathways to provide essential nutrients and molecules for cellular activity. Understanding these transport pathways is essential to maintaining and improving the health of articular cartilage and ultimately synovial joints. Solute transport has been studied extensively, and it has been established that solute size, shape, and charge all influence diffusion [30]-[37]. In addition, movement, specifically compression, has been shown to enhance the uptake of solutes into the cartilage matrix [33], [44], [49], [54]. The underlying mechanism has been attributed to the recovery of interstitial fluid lost during static loading.

Most studies that have examined the influence of load on solute uptake have focused primarily on uniaxial compression (cyclic or static). Only recently have the contributions of sliding, as it relates to solute transport, been considered despite it being the predominate component of joint motion. Burris et al. have studied the mechanisms of sliding-enhanced solute transport and demonstrated that solute accumulation, cartilage thickening, and friction reduction are all driven by a processes termed tribological rehydration [41], [42], [48]. While their work describes the occurring phenomenon, it does not provide a quantitative comparison to other loading modes. They studied a buried contact area to eliminate the effects of mechanical pumping due to dynamic compression; while this is useful for isolating the effect of pure sliding on solute uptake, its in vivo translation is limited due to the stationary contact location on the cartilage. Furthermore, previous studies, including those involving sliding, have employed loading modes with a static contact location. Lacking a quantitative comparison, it remains unclear what 
component of joint motion, sliding or cyclic uniaxial compression, is a larger driver of solute transport.

Nutrient supply and oxygen content [55] are inherently low in articular cartilage compared to other tissues and associated with the low metabolic activity of chondrocytes[56]. To transport solutes into the deeper regions of the tissue, solutes need to pass through the superficial region which has a lower permeability compared to the remaining tissue [57], [58]. The densely packed collagen fibers, oriented parallel to the articular surface, and increased fixed charge density have been attributed to this decreased permeability. While it is known that mechanical loading will have enhanced solute uptake compared to passive diffusion, it remains unclear if mechanical action is merely overcoming the low permeability of the superficial region or exceeding equilibrium potential of the tissue.

Therefore, the objectives of this study were two-fold: first, to compare solute uptake driven by sliding with a migrating contact area to typical cyclic uniaxial compression. And secondly, to evaluate the role of the superficial region on passive diffusion and compare to the results from loaded tests. To investigate this, we evaluated solute uptake in the absence of a superficial region. Primary outcome measures for this study were mass transfer, rate of mass transfer, and surface partition factor. We hypothesized that 1) uptake would be highest under sliding with a migrating contact area, followed by uniaxial compression, passive diffusion without the superficial zone and lastly intact passive diffusion. 2) Mass transfer induced by loaded conditions would exceed passive diffusion at equilibrium, and 3) Surface partition factors for loaded conditions would also exceed passive diffusion at equilibrium. 


\subsection{Materials and methods}

\subsubsection{Tissue preparation}

Porcine stifle joints were obtained from a local butcher and osteochondral plugs were harvested within 24 hrs of slaughter. At the time of slaughter the animals were approximately 6 months old and weighed between $113-118 \mathrm{~kg}$. Specimens were inspected for visible signs of damage to the articular surface prior to obtaining $10 \mathrm{~mm}$ diameter osteochondral plugs. The plugs were obtained from the patellofemoral groove and the patella using a coring drill bit. Approximately 8-10 plugs were obtained per joint. Plugs were submerged in phosphate buffered saline (PBS, $\mathrm{pH}$ 7.0) and frozen at $-20^{\circ} \mathrm{C}$ prior to use.

\subsubsection{Tracer preparation}

A single low molecular weight tracer, fluorescein sodium salt (Na-fluorescein, Sigma-Aldrich, MW $376 \mathrm{Da}$, anionic), with excitation/emission wavelengths of 494/513 nm was utilized for this study to mimic small nutrient molecules. The tracer was dissolved in PBS (pH 7.0) at a concentration of $0.01 \mathrm{mg} / \mathrm{ml}$.

\subsubsection{Diffusion tests}

Specimens were thawed to room temperature prior to diffusion experiments. Tests were performed under three types of conditions: continuous cyclic axial or sliding loading or unloaded passive diffusion. Based on pilot testing, samples were sealed in purpose made diffusion chambers to eliminate diffusion through the radial edge. Pilot testing indicated diffusion through the tissue in the transverse direction was faster compared to diffusion through the articular surface and was eliminated for this reason. In addition, diffusion through the articular surface is a 
more accurate assessment of in vivo conditions. The bone side of the chamber was filled with PBS (pH 7.0) to maintain tissue hydration while the cartilage side was filled with the fluorescent tracer.

Specimens were subjected to one of two possible loading conditions: cyclic uniaxial compression or cyclic sliding with a migrating contact area. For both configurations, a $25 \mathrm{~mm}$ diameter stainless steel spherical indenter was used. Sliding motion was applied across the articular surface using a custom apparatus, briefly, a ball screw operated by a stepper motor provided reciprocating motion and two transmissive optical encoders recorded indenter position in axial and transverse directions. A moving contact area was chosen to best represent physiological joint motions; sliding was maintained at $5 \mathrm{~mm} / \mathrm{s}$. For sliding tests, a static axial load of $1.6 \mathrm{~N}$ was applied. For the cyclic compression tests, a 1.6 N load amplitude was applied using an MTS Sintech $1 / \mathrm{G}$ fitted with a $5 \mathrm{~N}$ load cell in load control (Software Testworks Elite). Cycle frequency was $0.6 \mathrm{~Hz}$ for both loading modes. Loaded tests were maintained for $0.5,1$ and $2 \mathrm{hrs}$ at room temperature. The experimental setup is shown in Figure 2-1. 


\section{$1.6 \mathrm{~N}$ constant load}

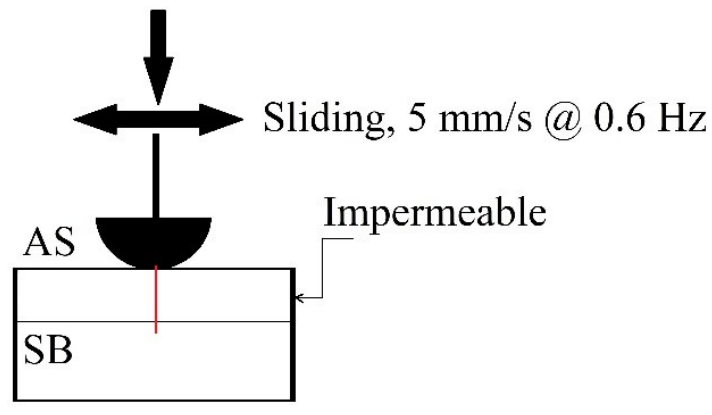

Cyclic uniaxial compression

$0-1.6 \mathrm{~N} @ 0.6 \mathrm{~Hz}$

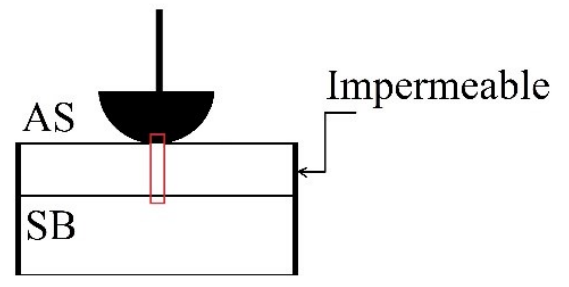

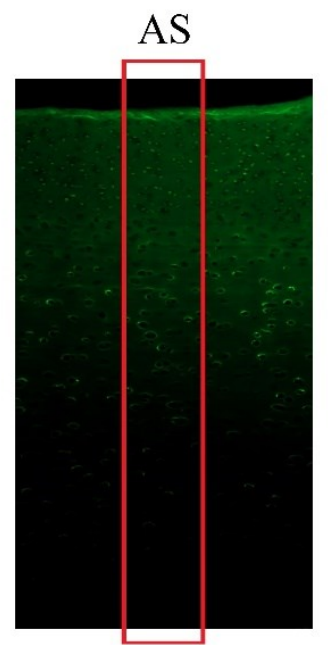

$\mathrm{SB}$

$10 \mathrm{~mm}$

Figure 2-1: Experimental setup for dynamically loaded tests. Both conditions were maintained at a cycle frequency of $0.6 \mathrm{~Hz}$ for $0.5,1$, and $2 \mathrm{hr}$ durations. The radial edge of the tissue was sealed to restrict solute transport through the articular surface only. For samples subject to sliding, sections were obtained perpendicular to sliding direction. $\mathrm{SB}=$ subchondral bone, $\mathrm{AC}=$ articular surface

Specimens were subject to one of three types of passive diffusion conditions: intact, diffusion following removal of the superficial zone or diffusion following lubricin extraction. These conditions were chosen to approximate healthy, physical injury, and early degeneration, respectively. Superficial zone removal of the first $100 \mu \mathrm{m}$ of the tissue was performed using a microtome cryostat (Microm HM-500M) allowing for precise sectioning of the tissue and preventing the need for an additional freeze thaw cycle. Lubricin extraction was performed according to the methods of Jones et al. by incubating samples in a concentrated salt solution (PBS containing 1.5 M NaCl) for 20 minutes [59]. Diffusion for intact samples was observed for 
12, 24 and $72 \mathrm{hrs}, 12,24$, and $72 \mathrm{hrs}$ for specimens with the superficial zones removed, and 6, 12, and $24 \mathrm{hrs}$ for specimens with lubricin extracted. Experimental times for each group were chosen based on the time to reach equilibrium, and the concentration of fluorescent tracer that could be imaged without exceeding the dynamic range of the sensor. Time points for loaded and passive conditions differed since solute transport in unloaded specimens was negligible at the time points used to study load-induced diffusion.

\subsubsection{Immunohistochemical assessment of lubricin extraction}

To validate the removal of lubricin using the methods previously described [59], 10\% formalin fixed cartilage plugs were probed for lubricin using whole mount techniques. Briefly, samples were incubated in $1 \mathrm{X}$ tris-buffered saline (TBST), treated with 3\% hydrogen peroxide blocking solution, and probed with monoclonal mouse anti-Lubricin/Proteoglycan 4 Clone 9G3 antibody (Millipore/Sigma MABT401) before detection using 3,3'diaminobenzidine chromogen substrate kit (Abcam, ab64238).

\subsubsection{Fluorescent imaging and concentration profiles}

Following diffusion tests, samples were snap frozen and $18 \mu \mathrm{m}$ cross-sectional cuts were taken perpendicular to the bearing surface. Samples were imaged immediately using a Zeiss AxioImager M2 epifluorescent microscope under 5x magnification with a filter for Nafluorescein (Figure 2-2). Focusing and sample orientation were done under low power transmitted white light to prevent photobleaching. Intensity profiles were mapped along the full depth of the tissue (articular surface to subchondral bone) by averaging across a $50 \mu \mathrm{m}$ wide region of interest. Fluorescent intensity was converted to a concentration using a standard curve generated by serial dilutions (Appendix A:). 


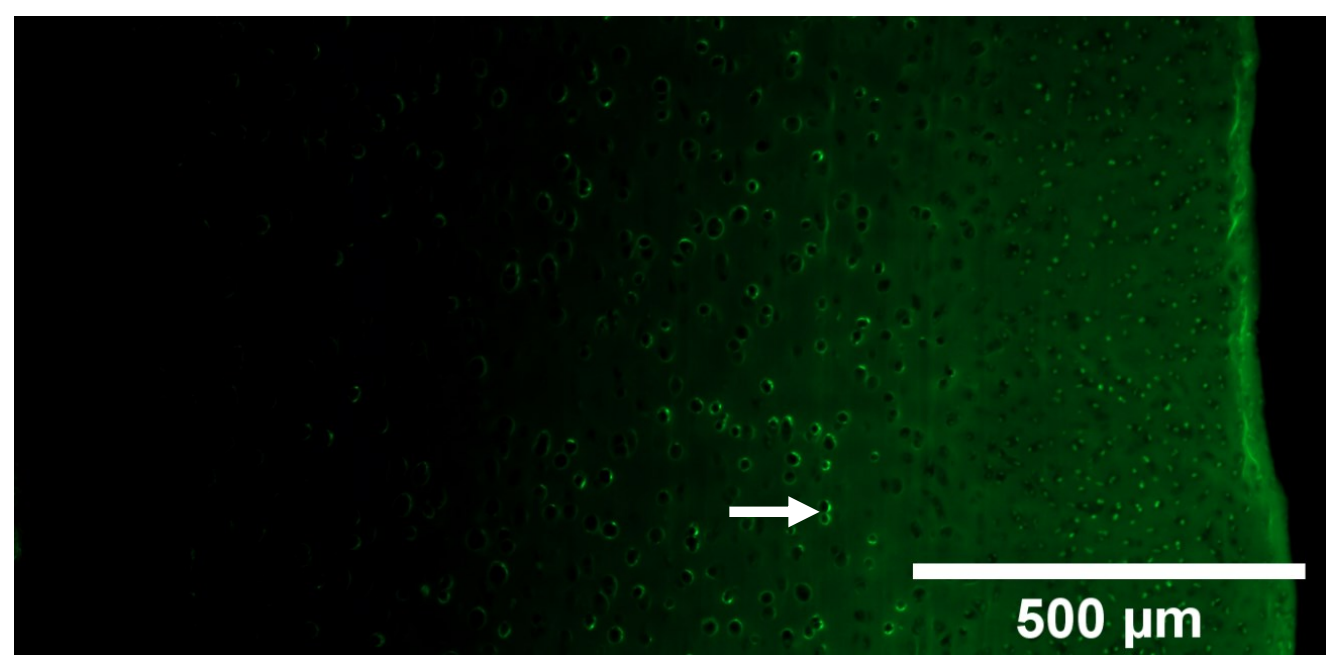

Figure 2-2: Fluorescent cross-sectional image of porcine articular cartilage following 2 hrs of sliding with a migrating contact area. Image was obtained using an epifluorescence microscope under $5 \mathrm{X}$ magnification with a filter for fluorescein. Tracer accumulation in the pericellular matrix can be seen as bright spots around chondrocyte lacunae (arrow); concentration profiles are spatially averaged at each depth location and include these features.

\subsubsection{Mass transfer}

Total mass transfer and the rate of mass transfer per unit surface area were determined through numerical integration of the concentration profile using a trapezoidal method.

\subsubsection{Surface partition factor}

A surface partition factor was calculated using the methods of Torzilli et al. to describe the fluidcartilage interface [50]. This characterizes the jump boundary condition that occurs because of the difference in solute concentration between the bath and the tissue:

$$
K=\frac{C\left(l_{0}, t\right)}{C_{0}}
$$

where $C\left(l_{0}, t\right)$ is the concentration at the surface and $C_{0}$ is the concentration in the bath. 


\subsubsection{Statistical analysis}

All data is presented as a mean with $95 \%$ confidence intervals. Where necessary for graphical clarity, only the positive bound of the confidence interval is displayed. Concentration profiles were compared over the entire the depth of the tissue using statistical parametric mapping [60]. Comparisons between total mass transfer, rate of mass transfer, and surface partition factors were done using a Mann Whitney U test for non-parametric data (IBM SPSS Statistics 23).

Significance was set to 0.05 for all tests. Sample sizes varied between groups and time points and are outlined in the results section.

\subsection{Results}

\subsubsection{Observations between loaded conditions}

There was visible tissue consolidation for all samples subject to cyclic uniaxial compression. Tissue consolidation was not visibly observed for sliding and it has been shown that it is negligible in this loading mode [61].

\subsubsection{Concentration profiles}

Passive concentration profiles had a characteristic middle region (between $20-80 \%$ of the tissue depth) with higher solute accumulation compared to the superficial and deep zones (Figure 2-3). This shape was less pronounced in loaded specimens although the characteristic middle region was beginning to develop at $2 \mathrm{hrs}$ for loaded conditions. 



Figure 2-3: Mean concentration profiles along the normalized length for (A) surface removed (B) intact. Shaded region shows $\mathbf{9 5 \%}$ confidence interval of the mean. For the intact condition, samples sizes were 4, 7, and 6 for $12 \mathrm{hr}, 24 \mathrm{hr}$, and $72 \mathrm{hr}$ timepoints respectively. Samples sizes were 3, 6, and $3 \mathrm{for} 12 \mathrm{hr}, 48 \mathrm{hr}$, and $72 \mathrm{hr}$ timepoints respectively for samples without a superficial region.

Solute uptake was higher in samples with the first $100 \mu \mathrm{m}$ removed compared to intact samples at equilibrium (Figure $2-3 ; \mathrm{p}=0.038$ ). There was no observable change between concentration profiles at 12,48 , and $72 \mathrm{hrs}$, indicating that in the absence of a superficial region, equilibrium was reached within the first 12 hrs. Of the loaded conditions, sliding had a significantly higher concentration within the tissue compared to uniaxial compression at all time points (Figure 2-4; $\mathrm{p}<0.001, \mathrm{p}=0.030, \mathrm{p}<0.001$ for $0.5,1$, and 2 hrs respectively). 

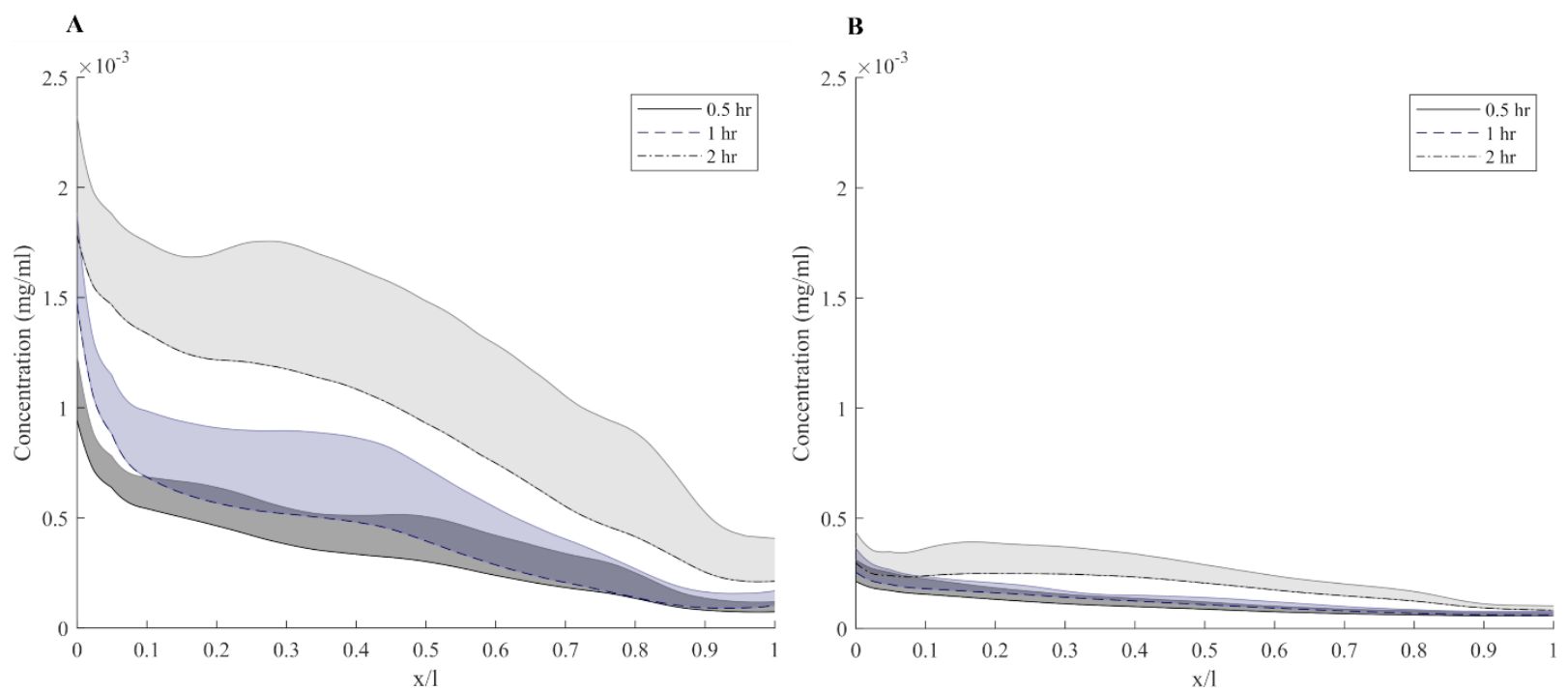

Figure 2-4: Mean concentration profiles along the normalized length for cyclic sliding (A) and cyclic compression $(B)$. Shaded region shows $+95 \%$ confidence interval of the mean. All timepoints had 6 specimens per group except for sliding at $1 \mathrm{hr}(\mathrm{n}=5)$.

\subsubsection{Mass transfer}

Total mass transfer (Figure 2-5) was 2.1-fold higher at $0.5 \mathrm{hr}$ for sliding compared to uniaxial compression $(\mathrm{p}=0.004)$. This increased to 2.6 -fold at $1 \mathrm{hr}(\mathrm{p}=0.032)$ and 4.4 -fold at $2 \mathrm{hrs}$ $(\mathrm{p}=0.002)$. Total mass transfer for both loading conditions at $2 \mathrm{hrs}$ had reached or exceeded intact passive diffusion at $12 \mathrm{hrs}, 1.34 \mathrm{E}-4(0.84 \mathrm{E}-4,1.84 \mathrm{E}-4) \mathrm{mg} / \mathrm{cm}^{2}$ and $3.08 \mathrm{E}-5$ (1.55E-5, $4.60 \mathrm{E}-5) \mathrm{mg} / \mathrm{cm}^{2}$ for sliding and uniaxial compression respectively compared to $2.69 \mathrm{E}-5$ (2.19E-5, 7.57E-5) $\mathrm{mg} / \mathrm{cm}^{2}$ for intact passive diffusion. 

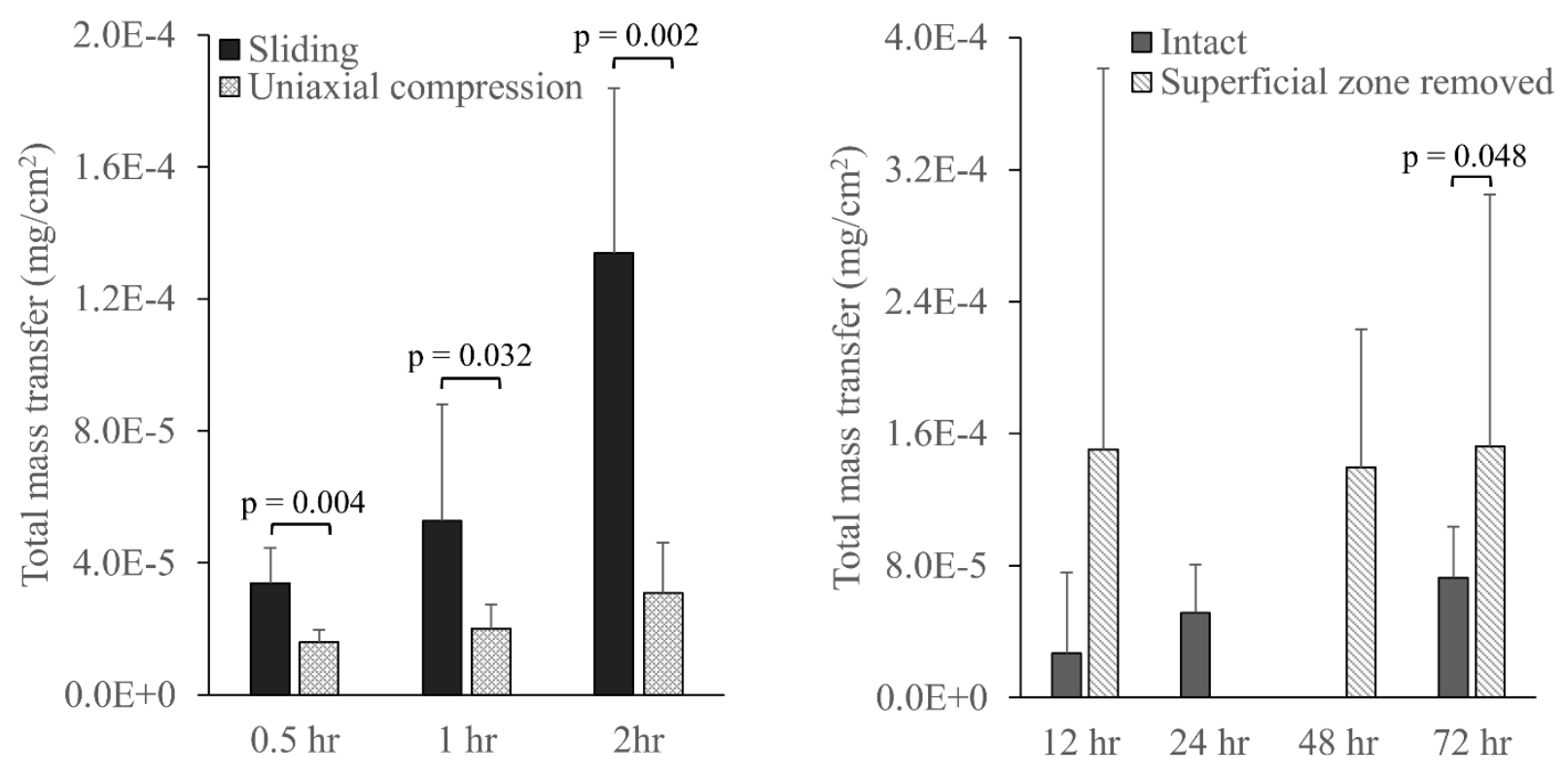

Figure 2-5: Mean mass transfer for all experimental groups and timepoints. Error bar shows $+95 \%$ confidence interval of the mean. Result of significant Mann Whitney $U$ tests are displayed on plots.

For samples subject to sliding, mass transfer rate was not significantly different between timepoints, mean mass transfer rate was 6.30E-5 (5.508E-5, 7.52E-5) $\mathrm{mg} / \mathrm{cm}^{2} \mathrm{hr}$ for all time points. Rate of mass transfer continuously decreased between time points for uniaxial compression indicating the tissue was reaching equilibrium (Figure 2-6). A similar trend was observed for both passive conditions. Total mass transfer and rate of mass transfer was higher in samples without the superficial region compared to intact samples at equilibrium. At $72 \mathrm{hrs}$ total mass was 7.27E-5 (4.18E-5, 1.04E-4) for intact samples compared to $1.5 \mathrm{E}-4$ (-3.18E-7, 3.05E-4) for samples without the superficial region, this difference was significant $(\mathrm{p}=0.048)$. 



Figure 2-6: Mean rate of mass transfer for all experimental groups and timepoints. Error bar shows $+95 \%$ confidence interval of the mean. Result of significant Mann Whitney $U$ tests are displayed on plots.

\subsubsection{Surface partition factors}

Surface partition factors increased with increasing experimental duration for intact and biaxial sliding specimens (Figure 2-7). For uniaxially loaded samples, there was a small increase between 0.5 - and 1-hr time points and no significant change between 1- and 2-hr time points $(\mathrm{p}=0.662)$. Surface partition factor was higher for sliding compared to uniaxial loaded samples at all time points $(\mathrm{p}=0.002, \mathrm{p}=0.008, \mathrm{p}=0.002$, for $0.5 \mathrm{hr}, 1 \mathrm{hr}$, and $2 \mathrm{hrs}$ respectively). By $2 \mathrm{hrs,}$ surface partition factor for sliding had reached $0.18(0.12,0.24)$ compared to $0.03(0.01,0.04)$ for uniaxially loaded samples. For samples without superficial zones there is no observable change in partition factors across time points, further indicating that the samples had reached equilibrium at $12 \mathrm{hrs}$. At equilibrium (72 hrs), the surface partition factor for intact samples was $0.06(0.03,0.09)$ compared to $0.11(0.07,0.15)$ for samples without a superficial region, this difference was significant ( $\mathrm{p}=0.048$ ). By $2 \mathrm{hrs,} \mathrm{surface} \mathrm{partition} \mathrm{factor} \mathrm{for} \mathrm{sliding} \mathrm{had} \mathrm{exceeded}$ 
intact samples at $72 \mathrm{hrs}(\mathrm{p}=0.002)$ and was comparable to samples without a superficial region $(p=0.077)$.
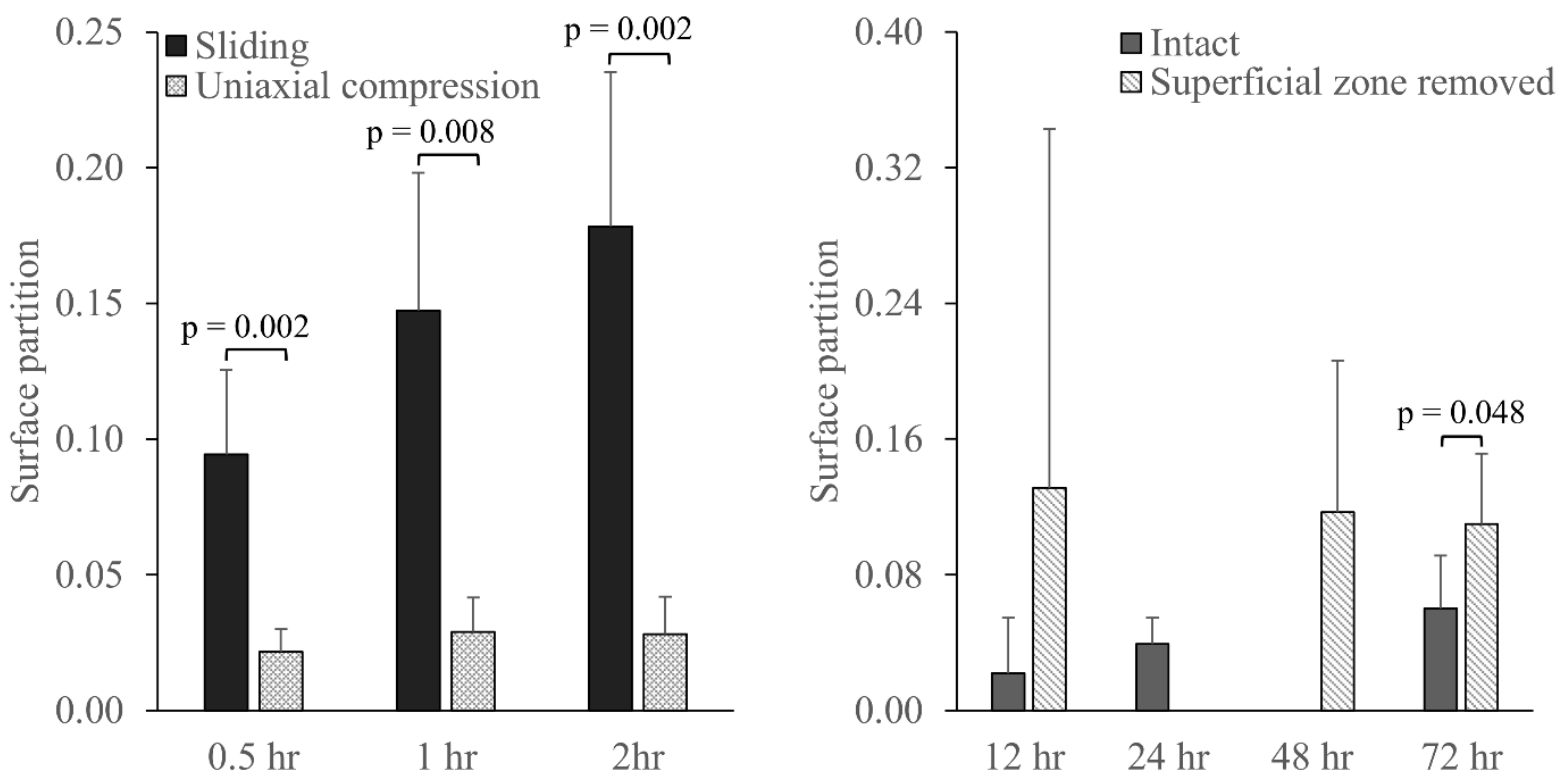

Figure 2-7: Mean surface partition factor for all experimental groups and timepoints. Error bar shows $+95 \%$ confidence interval of the mean. Result of significant Mann Whitney $U$ tests are displayed on plots.

\subsubsection{Immunohistochemical assessment of lubricin extraction}

Whole tissue samples were positive for lubricin indicating that the removal process was

unsuccessful. We believe this is due to the difference in tissue maturation between our specimens and those used by Jones et al. For this reason, the results are not discussed (See Appendix B:). The elevated solute uptake compared to intact specimens was likely a result of the changing charge density of the cartilage matrix following exposure to the high salt concentration rather than any physiologically relevant process. 


\subsection{Discussion}

For this study we used a negatively charged, low molecular weight solute to assess transport under loaded and passive conditions. The first goal of the study was to quantify mass transfer induced by a physiological motion (biaxial sliding with a migrating contact area). The results clearly demonstrate that physiological motion involving a moving contact location provides enhanced solute transport into and through the cartilage compared to uniaxial compression and passive diffusion. Total mass transfer was 4.4-fold higher for sliding compared to uniaxial loading after $2 \mathrm{hrs}$; mass transfer after $2 \mathrm{hrs}$ of sliding was approximately five-fold higher than passive diffusion at $12 \mathrm{hrs}$.

Mass transfer was chosen as the primary outcome for this study as it best characterizes the total amount of the fluorescent tracer within the tissue and is analogous to total nutrient or exogenous biochemical supply in vivo. Total mass transfer continues to increase until the samples are at equilibrium, while approaching equilibrium, rate of mass transfer decreases with increasing experimental duration. Surface partition factor was calculated to characterize the boundary interface between the tissue and the solute bath representing the permeability of the superficial region. In this study surface partition ranged from approximately $0.03-0.18$, which is lower than the surface partition factors reported by Torzilli et al. [50]. These differences could be explained by tissue type (porcine vs bovine), skeletal maturity, solute charge, shape and or stokes radius.

There are several limitations associated with the methods used in this study: first, we only considered a single cycle frequency and load for mechanical testing, it is unclear if the differences in solute transport observed for sliding and uniaxial compression would hold true if these parameters were varied. In addition, like most studies, we used a metal indenter for 
articulation against the surface, this does not imitate in vivo conditions. While we were able to demonstrate that without the superficial region, equilibrium solute uptake is increased and achieved sooner, we are unable to identify what aspects of the superficial region contribute to this.

Though it is the predominate component of joint motion, the contribution of sliding to solute uptake within the cartilage matrix has remained unquantified in existing literature until recently [41], [42]. This study offers the first direct comparison of solute uptake induced by sliding with moving contact location and uniaxial compression. While both loading conditions reached or exceeded the total mass transfer for passive controls at $12 \mathrm{hrs}$, sliding resulted in significantly higher mass transfer and did not appear to be reaching equilibrium at $2 \mathrm{hrs}$. At $2 \mathrm{hrs,} \mathrm{the} \mathrm{surface}$ partition factor for sliding was approximately 6.3 times higher than uniaxial compression. There are several mechanisms that could explain the enhanced uptake with sliding, namely, tribological rehydration, and enhanced fluid flow across the surface and within the tissue [62]. Since tribological rehydration is the fundamental mechanism behind sliding-induced solute transport [41], [42], this likely accounts for most of the difference. Tribological rehydration is a result of elasto-hydrodynamic pressurization driving fluid into the surface at a rate equal to the rate of fluid exudation caused by interstitial fluid pressurization. In our study we utilized a migrating contact area which would have the added advantage of mechanical pumping induced by the changing contact area. During cyclic compression, tissue consolidation results due to the absence of a counterbalance to interstitial pressurization diving fluid out of the tissue [62].

The second goal of this study was to assess equilibrium solute transport with and without the superficial region to determine if load-induced diffusion overcomes the low permeability of the 
surface or exceeds passive capacity of the tissue. As anticipated, removing the superficial region of the tissue resulted in increased solute transport and decreased time to reach equilibrium. There are several constituents of the superficial region that could be acting as a barrier to diffusion: lubricin, the densely packed collagen network or proteoglycan content. By removing the superficial region, it is not possible to identify which constituent or combination of constituents is responsible for this result. We attempted to remove lubricin according to Jones et al. [59], although subsequent staining revealed lubricin remained at the surface. The increased diffusion following this treatment may have been due to altered charge density within the surface e.g. due to accumulation of counter-ions, allowing a higher transport rate of the anionic tracer.

By 2 hrs, samples subject to sliding were able to achieve mass transfer that occurs in the absence of a superficial region at equilibrium. Considering the rate of mass transfer was not declining, it is likely that solute uptake induced by sliding would exceed passive tissue capacity and is therefore not just overcoming the low permeability of the superficial region. These results suggest that sliding may be a useful mechanism for driving nutrients and growth factors into engineered constructs to enhance tissue composition and properties. Several studies have noted depth dependent spatial gradients of extracellular matrix components with concentrations decreasing with distance from the media exposed surface [63]-[65]. Thus, sliding could enhance matrix composition in engineered tissues, especially in the deep zone, due to increased supply of nutrients and growth factors.

This study provides the first evidence that solute uptake under sliding motion exceeds that of cyclic uniaxial compression. In addition, sliding is not just overcoming the low permeability of the superficial zone, it is inducing solute transport beyond the passive capacity of the tissue. This 
is an important finding and the implications of this pathway need to be considered, specifically as it relates to biochemical signaling, nutrient transport, drug delivery, and engineered cartilage. 


\section{Multilayer nutrient diffusion modeling in articular cartilage subject to sliding and uniaxial compression}

In Preparation: Journal of Osteoarthritis and Cartilage 


\subsection{Abstract}

Objectives: The objectives of this study were: first, to compare the accuracy of single and multilayer nutrient diffusion models for representing load induced diffusion in articular cartilage. Second, evaluate depth dependent diffusivity using a multilayer model following dynamic load.

Design: Tests were performed on osteochondral plugs under two types of dynamic conditions: sliding and uniaxial compression. The articular surfaces were exposed to a fluorescent bath and solute uptake was quantified from the surface to the subchondral bone using fluorescent microscopy. Concentration profiles were fit to single, and multilayer diffusion models for all experimental timepoints. The multilayer model was then used to assess depth wise diffusivity. Primary outcome measures were RMSE for assessing model fits and diffusion coefficient for comparing spatiotemporal changes to diffusivity induced by dynamic load.

Results: Diffusivity varied depth-wise from the articular surface for both loading modes; as a result, concentration profiles were best characterized using a multilayer model. The RMSE of the fits obtained for sliding and uniaxial compression were respectively 5.7-fold and 3.9-fold better for the multilayer model compared to the single layer model. For samples subject to sliding, peak diffusivity shifted superficially and increased with increasing experimental duration.

Conclusions: These results are the first to quantify depth wise spatiotemporal changes to diffusivity under dynamic load in articular cartilage. The findings provide means for further modelling of solute transport in healthy and in changing tissue, either due to degeneration or evolving cartilage in tissue engineering.

Keywords: solute transport; sliding contact; axial loading, diffusion modeling, multilayer 


\subsection{Introduction}

In avascular tissues such as articular cartilage, diffusion is the primary mode of solute transport required for normal health and function. The diffusion coefficient, $D$, is used to characterize diffusivity and is a function of tissue composition and the diffusing solute. Characterizing diffusion is important in articular cartilage as it is well understood that the ability of a solute to diffuse through the tissue will impact therapies for osteoarthritis [66]. Rate of diffusion into the tissue is especially important due to the clearance time of therapeutics from synovial fluid [67]. Solute transport is also critical for nutrient supply and biochemical signalling to chondrocytes, the cells responsible for maintaining cartilage tissue [68], [69]. For this reason, molecular diffusion through cartilage has been an active area of study.

The composition and structure of articular cartilage varies depth-wise within the tissue. Overall, cartilage is characterized by three distinct regions: superficial, middle, and deep. In the superficial region the collagen network is oriented parallel to the bearing surface, this transitions to an oblique orientation in the middle region and perpendicular in the deep region. In addition to the collagen network, GAGs and water constitute a large portion of the tissue content. In healthy tissue, GAG content increases as a function of depth from the articular surface corresponding to an increase in fixed charge density and decreasing pore size [15].

Several studies have examined how local composition influences diffusivity [33], [38]-[40], [46]. Leddy et al. found that diffusivity was non-uniform between the various regions of articular cartilage. Interestingly, the pattern was not consistent for various sized dextrans [38]. Their research goes on to explain these findings through characterizing diffusional anisotropy in collagenous tissues [39]. They found that diffusion is anisotropic in these types of tissues with 
the highest rates along the primary orientation of the fibers. In addition, there is a size dependency to diffusion anisotropy whereby small solutes will exhibit little interaction with widely-spaced collagen fibers, explaining their earlier findings [38]. More recently DiDomineco et al. studied antibody diffusion through the articular surface of healthy and enzymatically degraded cartilage. Their study found that spatial-dependent diffusivities are related to local structure and composition and were able to demonstrate that the highly aligned collagen at the surface acts as a barrier to solute transport [40]. These authors also looked at the effect of varying strain amplitudes on depth-dependent diffusivity. They found significant enhancement $275 \mu \mathrm{m}$ below the articular surface with maximal enhancement at higher strain amplitudes (5\%). The primary limitation of their work was the loading conditions that they employed as dynamic radial compression does not exist in vivo.

To our knowledge no study exists that has studied depth dependent diffusivity of articular cartilage subject to sliding. Therefore, the goals of this study were: 1) compare the accuracy of nutrient diffusion models (single vs multi-layer) for representing load induced diffusion and 2) to evaluate depth dependent diffusivity of articular cartilage following sliding and uniaxial compression. We hypothesise that 1) due to the heterogeneity of the tissue, a multilayer diffusion model will best describe load-induced diffusion for both types of loading modes. 2) sliding and dynamic compression will result in varying diffusivity with tissue depth, and this will differ between loading modes. Primary outcome measures to assess these hypotheses will be root mean squared error (RMSE), concentration profiles, and diffusivity. 


\subsection{Materials and methods}

\subsubsection{Specimen preparation}

Diffusion experiments were performed as previously described (See Chapter 2). Briefly, $10 \mathrm{~mm}$ diameter osteochondral plugs were excised from the patellofemoral groove of porcine stifle joints using a coring drill bit within $24 \mathrm{hrs}$ of sacrifice. All samples were visually inspected for

macroscopic defects prior to use. At time of slaughter animals were approximately 6 months old weighing between 113-118 kgs. Plugs were randomly distributed to experimental groups (sliding, compression,) and frozen in $\mathrm{PBS}(\mathrm{pH} 7.0)$ at $-20^{\circ} \mathrm{C}$ prior to use.

\subsubsection{Mechanical protocol}

Specimens were subjected to two types of conditions, cyclic sliding with a moving contact area, and cyclic uniaxial compression. In both configurations, a compressive $1.6 \mathrm{~N}$ load was applied using a $25 \mathrm{~mm}$ diameter stainless steel indenter at a cycle frequency of $0.6 \mathrm{~Hz}$. Sliding was applied across the articular surface using a custom-built apparatus and maintained at $5 \mathrm{~mm} / \mathrm{s}$ reciprocating over an $8 \mathrm{~mm}$ section. Cyclic compression with a $1.6 \mathrm{~N}$ amplitude was achieved using an MTS Sintech 1/G fitted with a 5 N load cell (MTS SMTI-5N-192, MTS Corp, USA; serial number E50840). The load apparatus was controlled with the manufacturer-supplied software (Software Testworks Elite). Tests were maintained for 0.5, 1 and $2 \mathrm{hrs}$ at room temperature ( $n=6$ per timepoint for all experimental groups except sliding at $1 \mathrm{hr}, \mathrm{n}=5$ ). During diffusion experiments, all specimens were sealed using quick setting epoxy into purposemade diffusion chambers to eliminate diffusion from the radial direction. The bone side of the chamber was filled with PBS (pH 7.0) and the cartilage side was exposed to a fluorescent solution (Figure 3-1). 


\subsubsection{Fluorescent tracer}

This study used a single low molecular weight anionic tracer (Na-fluorescein, Sigma-Aldrich, MW 376 Da, excitation/emission wavelengths of $494 / 513 \mathrm{~nm}$ ), dissolved in PBS (pH 7.0) at a concentration of $0.01 \mathrm{mg} / \mathrm{ml}$.
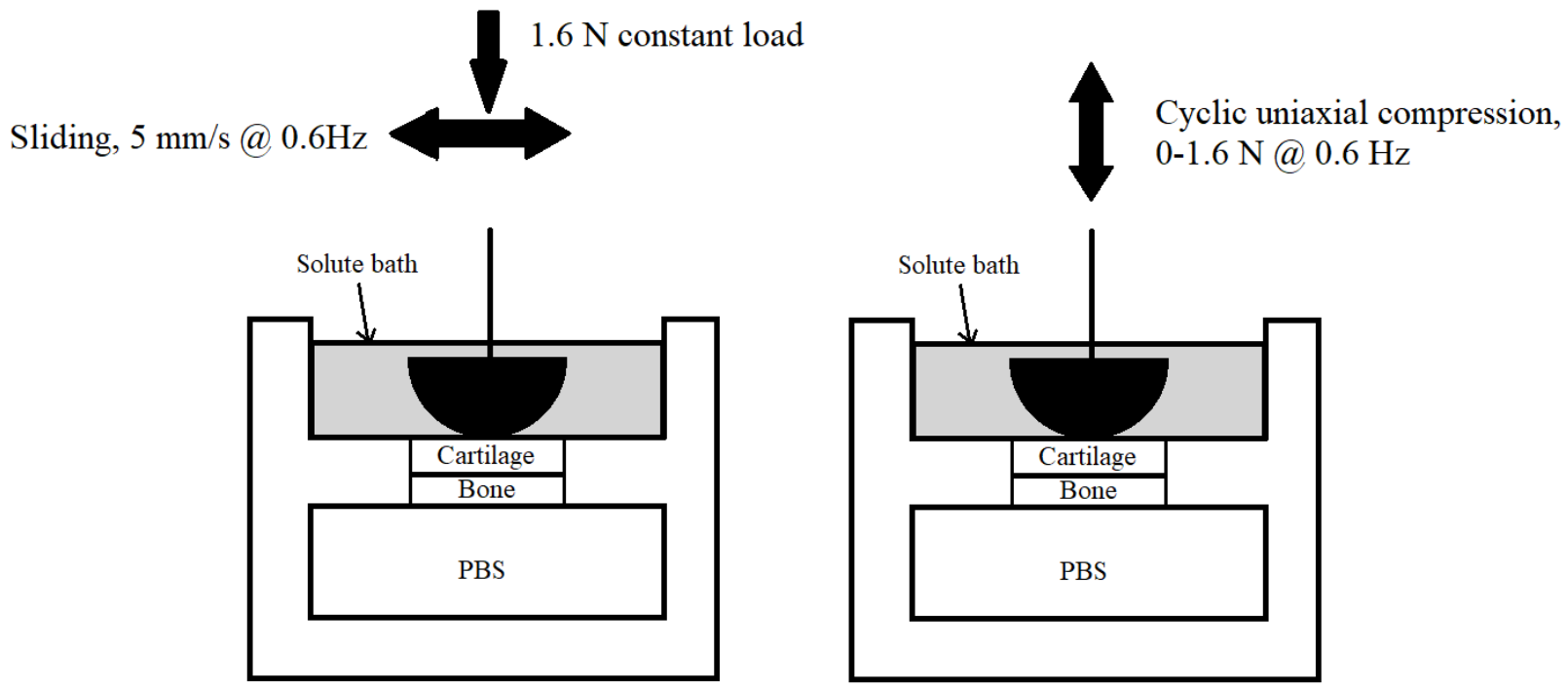

Figure 3-1: Cross sectional schematic of experimental set up. Osteochondral plugs were sealed around the radial edge to prevent solute uptake from this direction. Fluorescent tracer could only enter the tissue through the bearing surface. Arrows indicate direction of motion and dynamic loads: sliding motion (left) and uniaxial compressive force (right).

\subsubsection{Transport analysis}

Following diffusion experiments, samples were snap frozen and $18 \mu \mathrm{m}$ thick sections were cut perpendicular to the bearing surface on a freezing microtome. Sections were obtained from the central loaded region approximately $40-60 \mu \mathrm{m}$ apart. A minimum of 4 sections were obtained per osteochondral plug; the intensity profiles obtained from these sections were averaged to represent the result of a single sample. Samples were imaged immediately using a Zeiss AxioImager M2 epifluorescent microscope under 5x magnification with a filter for fluorescein. 
Focusing was done under low power transmitted light to prevent photobleaching. Microscope settings were chosen to ensure Gaussian distribution of the pixel intensities. Intensity profiles were generated from the surface to the subchondral bone averaging across a $50 \mu \mathrm{m}$ wide region of interest. Though minimal at the wavelength used (less than 1\%), tissue autofluorescence was determined and a correction was applied. Fluorescent intensity profiles were converted to a concentration using serial dilutions of the stock solution. The relationship between concentration and pixel intensity was linear in the ranges used for this study.

\subsubsection{One-dimensional transient diffusion models}

The experimental concentration profiles were fit to two numerical models of the transient onedimensional diffusion equation (Eq. 3-1).

$$
\frac{\partial C}{\partial t}=D_{e f f} \frac{\partial^{2} C}{\partial x^{2}}
$$

where $C$ is concentration, $t$ is time, $D_{\text {eff }}$ is the effective diffusivity that may include fluid flow effects, and $x$ is the spatial dimension. The first was a multilayer diffusion model developed by Carr and Turner [53] which allowed for multiple diffusivities to be calculated through the tissue depth. This model uses a vertex-centered finite volume method with a backward Euler discretization in time. Using this method, the concentration profiles were discretized into 20 equally-spaced layers; layers were assumed to be homogeneous with continuity of concentration between each layer (Figure 3-2). The number of layers was chosen based on a compromise between accuracy and computational time (Appendix C:). The second numerical model used the Crank-Nicolson discretization to solve the 1D diffusion equation, fitting a single diffusivity over the tissue depth. The Crank-Nicolson scheme was implemented in Matlab (Mathworks, Inc, 
2019a), with time steps set to $5 \%$ of the experimental duration and 1200 nodes equally spaced through the tissue depth. This model was adapted in our lab from Recktenwald [70]. These models are referred to as 'multilayer' and 'single layer', respectively. Experimental results were fit to both models using a least-squares minimization procedure (lsqcurvefit), that calculated the diffusivity value(s), D, that provided the smallest sum of squared differences between experimental data and simulation results using the built-in trust-region-reflective algorithm. The local solver (lsqcurevfit) was started from 20 different initial guesses of the diffusivity value to improve the likelihood of finding a globally optimal result.

Diffusivities calculated in this study are technically 'effective diffusivities' as they are not obtained under passive conditions, however for simplicity, the term 'diffusivity' will be used. Both models used the same boundary and initial conditions (Figure 3-2). At the tissue bath interface, the boundary concentration was set to equal the tissue concentration at the surface, $C(0, t)=C$, which was assumed constant. By doing this, the concentration discontinuity that exists between the bath and the tissue is accounted for. This occurs due to the surface partition factor being less than unity. This phenomenon was originally described by Torzilli et al. [50]. A zero-flux boundary condition was applied at the subchondral bone interface. 


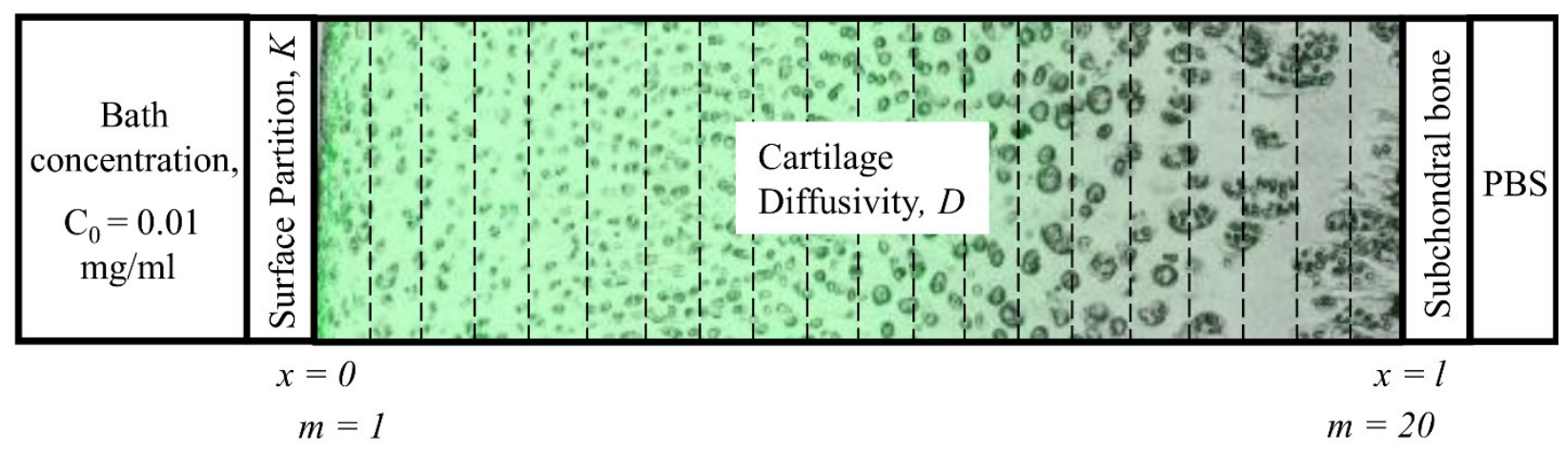

Figure 3-2: Schematic showing experimental conditions for multilayer diffusion model. $m$ indicates the layer number. Single layer is the same except the tissue is not subdivided into multiple layers as indicated by the dashed lines.

\subsubsection{Statistics}

All data is presented as a mean and $95 \%$ confidence intervals of the mean. Comparisons between outcomes at various time points was done using a Kruskal-Wallis H test (IBM SPSS Statistics 23). A Wilcoxon Signed-Rank Test was used to assess differences between multilayer and single layer model fits. Significance was set to 0.05 for all tests.

\subsection{Results}

Using the methods described, concentration profiles were generated for intact, healthy cartilage plugs subject to sliding and uniaxial compression. For all groups, mean cartilage thickness was 1589 [1432 - 1745] $\mu \mathrm{m}$. The concentration profiles for both loading modes indicate a heterogeneous diffusivity through the depth of the tissue. Fits obtained using a multilayer diffusion model were significantly better than the single layer model, coefficient of determination, was 0.993 [0.9899-0.9963] vs 0.3024 [-0.0323 - 0.6372] for multilayer and single layer respectively (Figure 3-3, Figure 3-4, $\mathrm{p}=0.028$ ). See Appendix D: for single layer and multilayer fits to individual specimens. 



Figure 3-3: Mean concentration profiles following 0.5, 1, and 2 hrs of biaxial sliding, shaded region represents $95 \%$ confidence interval of the mean. A) shows fit obtained using a least-squares approximation to the 1D transient diffusion model using a single layer model. B) shows fits obtained using a least-squares approximation to the 1D transient diffusion model using a multilayer model. To best visualize the difference between the multilayer and single layer models, the fits obtained for this figure were to the mean intensity profile at each time point. See Appendix B for results from individual specimens. 
A

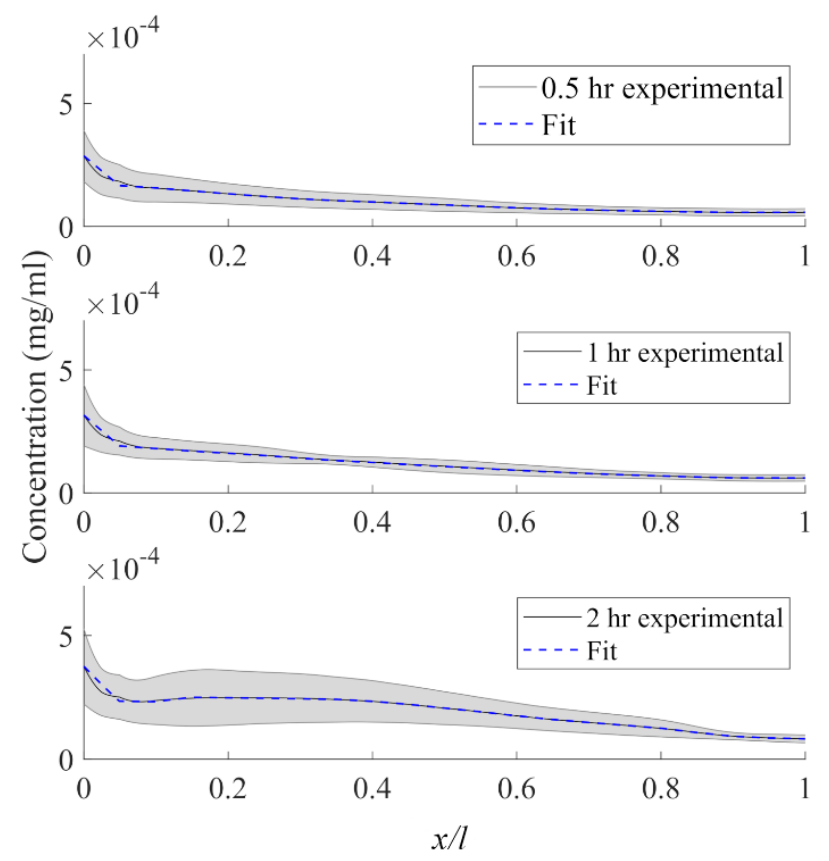

B



Figure 3-4: Mean concentration profiles for normalized depth (x/l) following 0.5 , 1, and 2 hrs of cyclic uniaxial compression, shaded region represents $95 \%$ confidence interval of the mean. A) shows fit obtained using a least-squares approximation to the 1D transient diffusion model using a single layer model. B) shows fits obtained using a least-squares approximation to the 1D transient diffusion model using the multilayer model. To best visualize the difference between the multilayer and single layer models, the fits obtained for this figure were to the mean intensity profile at each time point. 




Figure 3-5: Mean RMSE of experimental groups comparing fit obtained from multilayer and single layer models. Bar represents $95 \%$ confidence interval of the mean.

The RMSE of both experimental groups was significantly higher for the single layer model compared to the multilayer model further indicating that the heterogeneity of diffusivity within articular cartilage is best represented by a multilayer model (Figure $3-5, \mathrm{p}<0.001$ ). The RMSE of the fits obtained for sliding and uniaxial compression were 5.7-fold and 3.9-fold better for the multilayer model compared to the single layer model.

For both loading modes, at all time points, solute concentration decreased quickly in the first $10 \%$ of the tissue depth. This was followed by a plateau region for approximately $40-50 \%$ of the tissue depth. This region is most distinctive at longer time points for samples that had been subject to uniaxial compression. At $2 \mathrm{hrs,} \mathrm{this} \mathrm{region} \mathrm{appears} \mathrm{to} \mathrm{be} \mathrm{accumulating} \mathrm{solute} \mathrm{as} \mathrm{the}$ concentration is higher than in more superficial regions (Figure 3-4B).

For both loading modes studied, diffusivity varies as a function of tissue depth (Figure 3-6). Diffusivity was lowest in the first layer. For sliding there was no significant change in diffusivity 
for this layer with time $(\mathrm{p}=0.317)$. Mean diffusivity for all three time points was $11.5[1.51-$ 21.57] $\mu \mathrm{m}^{2} / \mathrm{s}$. For samples subject to dynamic compression diffusivity appeared to be decreasing with experimental duration, $41 \mu \mathrm{m}^{2} / \mathrm{s}$ at $0.5 \mathrm{hrs}$ to $14 \mu \mathrm{m}^{2} / \mathrm{s}$ at $2 \mathrm{hrs}$, however this difference was not significant $(\mathrm{p}=0.587)$. For samples subject to sliding, the highest diffusivity occurred in the plateau region (layers 3-11). This peak shifted superficially with increasing experimental duration, at $0.5 \mathrm{hrs}$ the peak occurred at layer 9, moving to layer 7 at $1 \mathrm{hr}$ and layer 5 at $2 \mathrm{hrs}$ (layer 1 is at the articular surface). Peak diffusivity also increased with increasing experimental duration, $319.6 \mu \mathrm{m}^{2} / \mathrm{s}$ at $0.5 \mathrm{hr}$ to $600.0 \mu \mathrm{m}^{2} / \mathrm{s}$ at $2 \mathrm{hrs}$. For all sliding timepoints, diffusion gradually decreased approaching the subchondral bone.

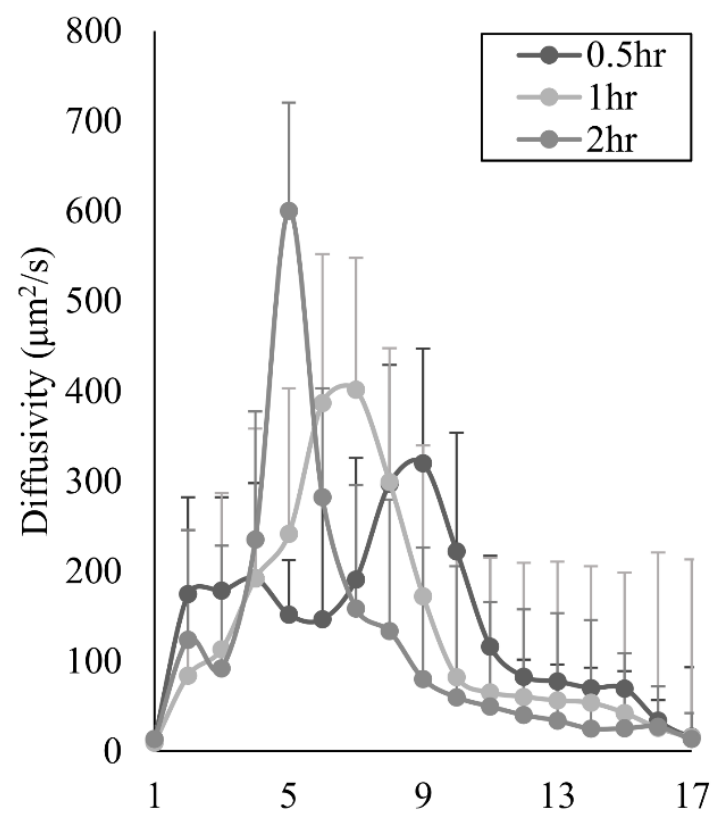

Layer



Layer

Figure 3-6: Diffusivity as a function of depth for samples subject to sliding (A) and cyclic compression (B). Diffusivity was determined using a least-squares approximation to the 1D transient multilayer diffusion model. Layers 18-20 are not shown due to the numerical errors associated with fitting a diffusivity in a plateau region of a concentration profile. 
For samples subject to dynamic compression, diffusivity varied as a function of tissue depth however diffusivity did not appear to follow the same trend as samples subject to sliding. Considering the first two timepoints, diffusivity was lower in all layers at $1 \mathrm{hr}$ of uniaxial compression compared to $0.5 \mathrm{hrs}$. For both timepoints, while diffusivity is generally higher in the plateau region, there are no characteristic peaks that were developed during sliding conditions. At 2 hrs, diffusivity within the plateau region is highest compared to earlier timepoints. At layer 3, "diffusivity" drops drastically to $-46.0[-139.7-48.0] \mu \mathrm{m}^{2} / \mathrm{s}$. This is due to the positive concentration gradient in this region (see Figure 3-4).

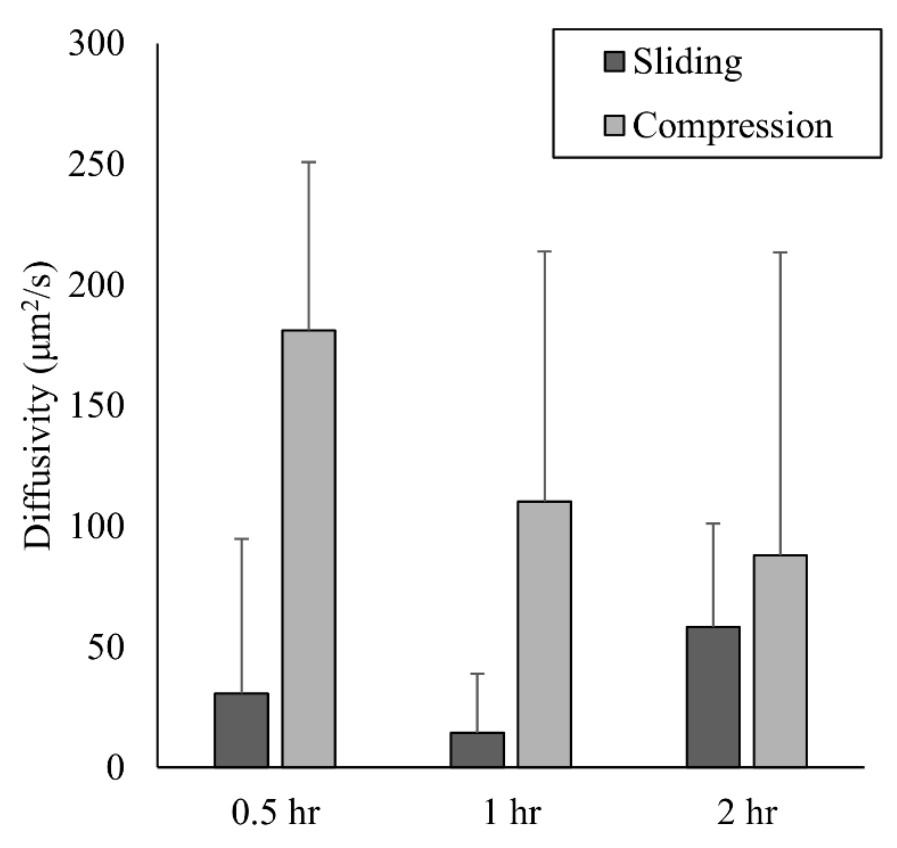

Figure 3-7: Diffusivity for experimental groups determined using a least-squares best fit to the 1D transient diffusion model. A single diffusivity was fit through the depth of the tissue.

Results from a single layer model show no significant difference in diffusivity across timepoints for samples subject to sliding (Figure 3-7; $\mathrm{p}=0.131$ ). For samples subject to dynamic 
compression, diffusivity appears to decrease with experimental duration, however this trend is not significant $(\mathrm{p}=0.710)$.

\subsection{Discussion}

The goals of this study were two-fold: to assess the accuracy of nutrient diffusion models at representing dynamic load induced diffusion in articular cartilage, specifically, for samples subject to sliding and uniaxial compression. The second goal of this study was to evaluate depthdependent diffusivity induced by these loading modes. This study showed that a multilayer model best characterizes the heterogeneous diffusivities within articular cartilage. In addition, this study showed that depth dependent diffusivity changes with time under loaded conditions. For samples subject to sliding, peak diffusivity shifted superficially and increased with increasing experimental duration.

The single layer diffusion model poorly characterized the concentration profiles observed in this study. This finding supports previous results which led to the use of the multilayer model developed by Carr and Turner [53] to characterize depth-dependent diffusion [33]. Subsequently, RMSE was approximately 5.7-fold lower for sliding and 3.9-fold lower uniaxial compression using the multilayer model.

Few studies have considered sliding as a driver of solute transport. Recent studies that have considered this loading mode were focused on various components of tribological rehydration (e.g. friction reduction, cartilage thickening, solute accumulation) and did not examine diffusivity. In addition, their work only considers the superficial region (first $230 \mu \mathrm{m}$ ) and therefore cannot provide any insight into the effect of sliding in deeper regions of the tissue. This 
is the first study that has looked at the spatiotemporal changes to diffusivity under sliding conditions.

While our loading conditions differed, the characteristic plateau region that we observed in our samples was also present in previous studies. The positive slope of the concentration gradient observed following $2 \mathrm{hrs}$ of cyclic compression appears to be an extension of the plateau region. This corresponded to increased diffusivities in our study and in previous work by other authors [33], [40], [46]. As raised by DiDomenico, one would expect highest diffusivities in the superficial regions [33], [38]. Our study, and several recent studies by DiDomenico et al., do not support this finding [33], [40], [46]. This might be due to differences in experimental methods. Studies that found higher diffusivity in the superficial region used FRAP techniques. This requires the tissue to reach equilibrium with the solute before disturbing it, and then measuring diffusivity during the return to equilibrium. Theoretically, diffusivity should be similar between techniques, however one distinct difference is that equilibrium is usually achieved via radial and axial transport with FRAP, which as demonstrated in Chapter 2, is not equivalent to equilibrium achieved strictly through the bearing surface. In addition, due to limitations with FRAP techniques, these experiments were all done under unloaded conditions.

There is significant depth-wise spatial variation in diffusivity for samples subject to dynamic load which changes with experimental duration. It should be noted that tissue consolidation is associated with uniaxial compression; this was observed in the loaded regions of our samples. Although not quantified, it has been previously documented [62] and would result in a decrease in the space available for molecular transport. For samples subject to sliding, tissue consolidation was not visible. This loading mode has been associated with cartilage thickening following static 
load [41], [42], [48]. In finite element simulations, sliding is associated with minimal tissue consolidation, whereas an average compressive creep response has been observed under cyclic uniaxial compression [62]. As water is exuded from the tissue matrix the tissue consolidates (a compositional change), resulting in decreased pore size and thus available space for solute migration. This might explain why the plateau region develops earlier for uniaxial compressive samples. If concentration were quantified per volume of available space instead of per volume of tissue, the concentration gradient might remain negative. Furthermore, quantification of solute concentration based on available space rather than tissue volume could also result in a monotonic, negative, concentration gradient to avoid the physically impossible negative diffusivity. Finite element analysis could be used in the future to study the porosity changes in time and space to estimate the effect on the diffusivity.

There are several limitations associated with the present work: first, like most studies that investigate load-induced solute transport, we use a metal-on-cartilage articulation as opposed to cartilage-on-cartilage, which does not imitate in vivo conditions. Secondly using diffusivity as the only metric for characterizing solute transport is misleading as total mass transfer is actually higher under sliding loads due to the increased surface partition factor (Chapter 2). The surface partition factor was found to be up to six-fold higher under sliding compared to uniaxial compression. In traditional analyses of diffusion through homogeneous media [52], this is equivalent to a bath concentration that is six-fold higher. The lower diffusivity values would otherwise seem to contradict the higher mass transfer (see Chapter 2) found with sliding compared to uniaxial loading. Diffusivity is limited to characterizing solute transport that is occurring strictly within the tissue and does not represent the system as a whole. However, in vivo molecular migration occurs from the intra-articular joint fluid across the cartilage surface 
and through the tissue; conversely, waste products must migrate in the opposite direction. Thus understanding in vivo mass transfer mechanisms in cartilage requires considering both the surface jump condition, quantified by the partition factor, as well as movement through the tissue, typically quantified by diffusivity.

The findings in this study provide means for further modelling of solute transport in healthy cartilage and in changing tissue, either due to degeneration or evolving cartilage in tissue engineering. These methods can be coupled with techniques for assessing depth wise compositional changes to tissue constitutes e.g. proteoglycan content through safranin-O staining which has been validated for natural and engineered cartilage [71]. Combining these methods would aid in understanding diffusivity changes during the degenerative process as proteoglycan loss is associated with early and mid-stage OA [72] and has been previously shown to alter bulk tissue diffusivity [31]. In addition, these methods could be used in future research to develop optimized loading in vivo to maximize nutrient/pharmaceutical delivery while maintaining low stresses on cartilage. This concept is known as 'continuous passive motion' and is a non-surgical treatment for knee OA [73]-[76]. The underlying mechanisms of its success remain unclear and the techniques used here could be used to investigate whether this is due to solute (nutrient, signalling) transport under low stress conditions. 


\section{Discussion and conclusions}


This work provides a comprehensive analysis of sliding induced solute transport in articular cartilage by considering mass transfer, surface partition, and diffusivity. Quantifying the uptake of a single low molecular weight tracer, sodium fluorescein salt, under loaded and passive conditions allowed the characterization of solute movement from the synovia to subchondral bone. The work presented in Chapter 2 demonstrated that sliding via a moving contact area enhances transport into articular cartilage, more so than cyclic uniaxial compression and passive transport. Total mass transfer was 4.4 -fold higher at $2 \mathrm{hrs}$ for sliding compared to uniaxial compression confirming hypothesis 1 , and approximately 5 -fold higher than passive transport at $12 \mathrm{hrs}$. Studying the surface layer demonstrated that not only is sliding overcoming the low partitioning of the superficial region, it has the potential to increase the concentration of the solute within the tissue beyond its passive capacity. After two hrs of sliding, total mass transfer had reached levels equivalent to specimens without the transport inhibiting superficial region confirming hypothesis 2 . Following the study of mass transfer and surface partitioning, the primary measures that characterize movement from the bath (synovia proxy) into the tissue, the spatial and temporal changes of diffusivity within the tissue were characterized. Due to the heterogeneous nature of articular cartilage, concentration profiles were best characterized when fit to a multilayer diffusion model that varies diffusivity as a function of depth confirming the $3^{\text {rd }}$ hypothesis. In addition, under sliding loads diffusivity increased with increasing experimental duration which was accompanied by a superficial shift in peak diffusivity. This trend was not observed for compression, confirming the $4^{\text {th }}$ hypothesis, that spatiotemporal changes to diffusivity would differ for sliding and uniaxial compression. 


\subsection{Limitations}

There are several limitations associated with the presented work. First, solute transport is only characterized for a single, nutrient sized solute. From previous work it has been established that solute transport is highly dependent on the shape, size, and charge of the diffusing solute [30][36], therefore it is hard to generalize these findings to other solutes without further experimental investigation. Larger molecules such as dextrans and antibodies would be required to understand the transport of biochemical signals such as cytokines and growth factors. This study utilized a small molecule to ensure some uptake when subject to passive conditions.

In addition, relatively short and continuous experimental durations were studied for loaded conditions. While these durations are within physiological ranges, they would typically be interrupted by intermittent rest and unloading. These studies also considered very low loads compared to in vivo, $1.6 \mathrm{~N}$ vs over $2000 \mathrm{~N}$ peak force for the hip or knee [77], [78]. However, during gait, loads are typically much lower during the swing phase when the most sliding occurs. Normal joint motion consists of various combinations of cartilage contacts (rolling, sliding, and uniaxial compression), therefore in vivo solute uptake during daily activity is likely a combination of the results presented in this study.

Furthermore, while the load application used in this study can be found in vivo, the interface condition, i.e. cartilage-on-metal cannot be. This boundary interface does not capture the electrostatic interactions that occur between surface proteins such as lubricin and may have an impact on diffusion. Finally, these tests were conducted at ambient temperature instead of physiological temperature $\left(37^{\circ} \mathrm{C}\right)$. It is expected that the lower temperature would result in decreased solute 
transport [79]. This would be consistent across experimental groups, although it may be less important in loaded specimens where fluid flow dominates mass transfer.

The experimental protocol used in this work subjected the osteochondral plugs to a single freezethaw cycle prior to use. This is common practice in cartilage research due to lengthy experimental protocols and difficulties obtaining fresh tissue at regular intervals; for these reasons, storage protocols are required. The effects of a single freeze-thaw cycle at $-20^{\circ} \mathrm{C}$ has been investigated previously and no significant deterioration to biomechanical or electromechanical properties was found [80]-[82]. Freeze-thaw cycles have the largest effect on chondrocyte viability in the absence of cryopreservation protocols [83], however, given the sparsity within the tissue, the effects on solute transport would likely be minimal and consistent between groups.

\subsection{Discussion}

The multiparameter characterization of solute transport used in this study provides a comprehensive understanding of in vivo solute transport into and through the depth of the tissue. Diffusivity is often studied independently in literature, however as shown in Chapters 2 and 3, that may be misleading due to surface partition which in this study was six-fold higher for sliding compared to uniaxial compression. By accounting for surface partition, the applied boundary conditions were not consistent between groups. This was necessary to prevent a concentration discontinuity between the solute bath and tissue. In most works [33], [40], [45], [46], [69], this is accounted for by normalizing tissue concentration to the surface and applying a common boundary condition of unity. As it relates to determining effective diffusivities, the methods are analogous. Once diffusivities are determined, changing the amount to solute partitioned from the 
bath will increase the concentration of solute within the tissue however the overall shape of the concentration gradient will remain similar for various surface partition factors.

Total mass transfer (or flux) is directly related to nutrient or biochemical signalling whereas diffusivity strictly characterizes movement within the tissue. An additional parameter that could be considered in future investigations is fluorescent tracer accumulation in the chondrocyte pericellular matrix (PCM) (Figure 2-2). This is accounted for in the calculation of total mass transfer although individually quantifying the tracer captured by the PCM may be a useful estimator of the amount of solute delivered to the chondrocytes and could be incorporated into existing mathematical models [84].

In Chapter 3 a negative diffusivity was observed following two hrs of cyclic uniaxial loading due to the positive slope of the concentration gradient. This is likely occurring due to the 'available space' for the solute to occupy within the ECM. The available space for solute transport exists within the pore space of the matrix, which is free water, as opposed to collagen-bound water in the intrafibrillar space. As the tissue consolidates, the pores collapse to exude water from the tissue; if factored in, the concentration gradient (i.e. per ml of available space) might stay negative. Universally, diffusion through articular cartilage is represented in terms of mass per volume of tissue rather than mass per volume of available space.

All passive transport conditions, and compressive samples at $2 \mathrm{hrs,} \mathrm{developed} \mathrm{a} \mathrm{positive}$ concentration profile in the middle region (Figure 2-3, Figure B-1). This appears to be an extension of the plateau region that develops at earlier time points, and has been observed in other studies [33], [40], [46]. The positive region might develop earlier under compression due to the accompanying tissue consolidation; almost no tissue consolidation is associated with sliding 
[62]. The change in the concentration gradient occurs within the first $10 \%$ of the tissue depth, in this region the tissue transitions from mostly organized collagen and water to randomly oriented collagen, water, and proteoglycan. The changing composition has a significant effect on the electrostatic environment and pore space; this change is not accounted for in the model used in this study. In addition, transport due to advection is incorporated in the effective diffusivity and not separately accounted for by the models used, however under loaded conditions it may be the dominant term in solute transport. Advection is challenging to assess without finite element models since is it is not constant, flow direction and magnitude change throughout the experiment and in general involves two- or three-dimensional flow fields even under uniaxial loads at the cartilage surface. Additionally, these contributions cannot be modeled in onedimension. Modeling solute transport as a two-dimensional process will likely improve the accuracy of the obtained results and should be considered in future work.

\subsection{Future research}

The work presented in this thesis provides a foundation for numerous future studies. As mentioned previously, solute shape, size, and charge all have significant impacts on the parameters characterized in this study. Investigating the effect of sliding on these parameters would be an important step towards developing therapeutics for OA. An extension of this would be investigating sliding-induced transport in OA specimens. It is possible due to the breakdown of the ECM and loss of proteoglycan associated with OA, that the enhancement to solute transport seen in healthy tissue might not translate to diseased tissue. The depth-dependent diffusivity model used in this study can be applied to diseased tissue or evolving cartilage in tissue engineering. Coupling tissue compositional changes i.e proteoglycan content through 
Safranin O staining [71], collagen fiber orientation through second harmonic generation microscopy (SHG) [85] would aid in understanding diffusivity changes during the degenerative process.

Most importantly, these methods have direct translation to clinical research and could be used in future research to develop optimized loading in vivo to maximize nutrient/pharmaceutical delivery while maintaining low stresses on cartilage. The isolated loading modes used in this study quantify individual contributions to solute transport in cartilage. Using this foundation, mapping solute transport within a whole joint through in situ simulators to mimic native joint motion [69] will be essential to translating these results to clinical practice.

A concept called 'continuous passive motion' is a non-surgical treatment for knee OA that has had clinical success although the underlying mechanism is unclear [73]-[76], [86]. The techniques used here could be used to investigate whether this is due to solute (nutrient, signalling) transport under low stress conditions. Finally, while we attempted to investigate the effect of lubricin deficiencies on solute transport, ultimately, we were unsuccessful, and this remains an important area of work yet to be studied. An effective method for removing lubricin molecules from the surface would be required.

\subsection{Contributions to the field}

The research presented in this thesis has made several important and novel contributions to understanding of solute transport in articular cartilage. We demonstrated that: 
- Sliding motion has the potential to greatly increase delivery of nutrients, biochemical signals and pharmaceuticals to the cartilage, enhancing tissue health/healing in patients, and ECM evolution in engineered cartilage.

- The low partition of the superficial region restricts the flow of nutrients into the tissue in unloaded conditions, this can be overcome by dynamic load, with the most enhancement achieved by sliding.

- Sliding is capable of inducing transport beyond the tissue's passive capacity.

- The multiparameter approach to characterizing solute transport in articular cartilage demonstrates that diffusivity, one of the most common and often sole parameters used to measure solute transport, does not accurately convey the movement of molecules from synovia to subchondral bone. Mass transfer, surface partition, and diffusivity are all needed to accurately characterize solute transport into and through the tissue.

- Experimental and simulation techniques were developed that could be used in future research to optimize rehabilitation protocols following cartilage injury or mild degeneration. 


\section{References}

[1] D. E. T. Shepherd and B. B. Seedhom, "Thickness of human articular cartilage in joints of the lower limb," Ann Rheum Dis, vol. 58, pp. 27-34, 1999.

[2] B. L. Wong, W. C. Bae, K. R. Gratz, and R. L. Sah, "Shear deformation kinematics during cartilage articulation: Effect of lubrication, degeneration, and stress relaxation," $M C B$ Mol. Cell. Biomech., vol. 5, no. 3, pp. 197-206, Sep. 2008.

[3] R. J. Goekoop et al., "Determinants of absence of osteoarthritis in old age," Scand. J. Rheumatol., vol. 40, no. 1, pp. 68-73, Jan. 2011.

[4] M. Lotz and R. F. Loeser, "Effects of aging on articular cartilage homeostasis," Bone, vol. 51, no. 2, pp. 241-248, Aug. 2012.

[5] V. C. Mow, S. C. Kuei, W. M. Lai, and C. G. Armstrong, "Biphasic creep and stress relaxation of articular cartilage in compression: Theory and experiments," J. Biomech. Eng., vol. 102, no. 1, pp. 73-84, 1980.

[6] A. Maroudas, "Balance between swelling pressure and collagen tension in normal and degenerate cartilage," Nature, vol. 260, no. 5554, pp. 808-809, Apr. 1976.

[7] M. Venn and A. Maroudas, "Chemical composition and swelling of normal and osteoarthrotic femoral head cartilage. I. Chemical composition," Ann. Rheum. Dis., vol. 36, no. 2, pp. 121-129, 1977.

[8] E. S. Oswald, P.-H. G. Chao, J. C. Bulinski, G. A. Ateshian, and C. T. Hung, "Dependence of Zonal Chondrocyte Water Transport Properties on Osmotic Environment," Cell. Mol. Bioeng., vol. 1, no. 4, pp. 339-348, Dec. 2008.

[9] P. A. Torzilli, "Influence of cartilage conformation on its equilibrium water partition," $J$. Orthop. Res., vol. 3, no. 4, pp. 473-483, 1985.

[10] F. C. Linn and L. Sokoloff, "Movement and composition of interstitial fluid of cartilage," Arthritis Rheum., vol. 8, no. 4, pp. 481-494, Aug. 1965.

[11] M. D. Grynpas, D. R. Eyre, and D. A. Kirschner, "Collagen type II differs from type I in native molecular packing," Biochim. Biophys. Acta - Protein Struct., vol. 626, no. 2, pp. 346-355, Dec. 1980.

[12] J. Yin, Y. Xia, and M. Lu, "Concentration profiles of collagen and proteoglycan in articular cartilage by Fourier transform infrared imaging and principal component regression," Spectrochim. Acta - Part A Mol. Biomol. Spectrosc., vol. 88, pp. 90-96, Mar. 2012.

[13] R. J. Minns and F. S. Steven, "The collagen fibril organization in human articular cartilage.," J. Anat., vol. 123, no. Pt 2, pp. 437-57, Apr. 1977.

[14] R. C. Lee, E. H. Frank, A. J. Grodzinsky, and D. K. Roylance, "Oscillatory Compressional Behavior of Articular Cartilage and Its Associated Electromechanical Properties," $J$.

Biomech. Eng., vol. 103, no. 4, pp. 280-292, Nov. 1981. 
[15] S. S. Chen, Y. H. Falcovitz, R. Schneiderman, A. Maroudas, and R. L. Sah, "Depthdependent compressive properties of normal aged human femoral head articular cartilage: relationship to fixed charge density," Osteoarthr. Cartil., vol. 9, pp. 561-569, 2001.

[16] J. Seror, L. Zhu, R. Goldberg, A. J. Day, and J. Klein, "ARTICLE Supramolecular synergy in the boundary lubrication of synovial joints," Nat. Commun., 2015.

[17] A. C. Bakker et al., "Overexpression of active TGF-beta-1 in the murine knee joint: Evidence for synovial-layer-dependent chondro-osteophyte formation," Osteoarthr. Cartil., vol. 9, no. 2, pp. 128-136, 2001.

[18] S. R. McGlashan et al., "Mechanical loading modulates chondrocyte primary cilia incidence and length," Cell Biol. Int., vol. 34, no. 5, pp. 441-446, 2010.

[19] D. D. D'lima, S. Hashimoto, P. C. Chen, C. W. Colwell, and M. K. Lotz, "Human chondrocyte apoptosis in response to mechanical injury," Osteoarthr. Cartil., vol. 9, no. 8, pp. 712-719, Nov. 2001.

[20] S. H. Elder, S. W. Sanders, W. R. McCulley, M. L. Marr, J. W. Shim, and K. A. Hasty, "Chondrocyte response to cyclic hydrostatic pressure in alginate versus pellet culture," $J$. Orthop. Res., vol. 24, no. 4, pp. 740-747, 2006.

[21] G. Candiani, M. T. Raimondi, R. Aurora, K. Laganà, and G. Dubini, "Chondrocyte Response to High Regimens of Cyclic Hydrostatic Pressure in 3-Dimensional Engineered Constructs," Int. J. Artif. Organs, vol. 31, no. 6, pp. 490-499, Jun. 2008.

[22] Health Canada, "Arthritis in Canada. An Ongoing Challenge," Ottawa, 2003.

[23] A. E. Peters, R. Akhtar, E. J. Comerford, and K. T. Bates, "The effect of ageing and osteoarthritis on the mechanical properties of cartilage and bone in the human knee joint," Sci. Rep., vol. 8, no. 1, Dec. 2018.

[24] S. Nakamura et al., "Changes in viscoelastic properties of articular cartilage in early stage of osteoarthritis, as determined by optical coherence tomography-based strain rate tomography," BMC Musculoskelet. Disord., vol. 20, no. 1, Sep. 2019.

[25] D. L. Robinson, M. E. Kersh, N. C. Walsh, D. C. Ackland, R. N. de Steiger, and M. G. Pandy, "Mechanical properties of normal and osteoarthritic human articular cartilage," $J$. Mech. Behav. Biomed. Mater., vol. 61, pp. 96-109, Aug. 2016.

[26] S. Inerot, D. Heinegard, L. Audell, and S.-E. Olsson, "Articular-Cartilage Proteoglycans in Aging and Osteoarthritis," Biochem. J, vol. 169, pp. 143-156, 1978.

[27] W. S. Hwang, B. Li, L. H. Jin, K. Ngo, N. S. Schachar, and G. N. F. Hughes, "Collagen fibril structure of normal, aging, and osteoarthritic cartilage," J. Pathol., vol. 167, no. 4, pp. 425-433, Aug. 1992.

[28] A. P. Hollander, I. Pidoux, A. Reiner, C. Rorabeck, R. Bourne, and A. R. Poole, "Damage to type II collagen in aging and osteoarthritis starts at the articular surface, originates around chondrocytes, and extends into the cartilage with progressive degeneration," $J$. Clin. Invest., vol. 96, no. 6, pp. 2859-2869, 1995. 
[29] X. Li et al., "In vivo T1rho and T2 mapping of articular cartilage in osteoarthritis of the Knee Using 3 Tesla Mri," Osteoarthr. Cartil., vol. 15, no. 7, pp. 789-797, 2007.

[30] A. Maroudas, "Transport of solutes through cartilage: permeability to large molecules.," $J$. Anat., vol. 122, no. Pt 2, pp. 335-47, Nov. 1976.

[31] P. A. Torzilli, J. M. Arduino, J. D. Gregory, and M. Bansal, "Effect of proteoglycan removal on solute mobility in articular cartilage," J. Biomech., vol. 30, no. 9, pp. 895-902, Sep. 1997.

[32] H. C. Chin, M. Moeini, and T. M. Quinn, "Solute transport across the articular surface of injured cartilage," Arch. Biochem. Biophys., vol. 535, no. 2, pp. 241-247, 2013.

[33] C. D. Didomenico et al., "The Effect of Antibody Size and Mechanical Loading on Solute Diffusion Through the Articular Surface of Cartilage," J. Biomech. Eng., vol. 139, no. 9, 2017.

[34] A. Maroudas, "Distribution and diffusion of solutes in articular cartilage.," Biophys. J., vol. 10, no. 5, pp. 365-79, May 1970.

[35] A. G. Bajpayee, C. R. Wong, M. G. Bawendi, E. H. Frank, and A. J. Grodzinsky, “Avidin as a model for charge driven transport into cartilage and drug delivery for treating early stage post-traumatic osteoarthritis," Biomaterials, vol. 35, no. 1, pp. 538-549, 2014.

[36] L. Zhang and A. Z. Szeri, "Transport of neutral solute in articular cartilage: Effects of loading and particle size," Proc. R. Soc. A Math. Phys. Eng. Sci., vol. 461, no. 2059, pp. 2021-2042, 2005.

[37] B. Sterner, M. Harms, S. Wöll, M. Weigandt, M. Windbergs, and C. M. Lehr, "The effect of polymer size and charge of molecules on permeation through synovial membrane and accumulation in hyaline articular cartilage," Eur. J. Pharm. Biopharm., vol. 101, pp. 126136, Apr. 2016.

[38] H. A. Leddy and F. Guilak, "Site-Specific Molecular Diffusion in Articular Cartilage Measured using Fluorescence Recovery after Photobleaching," Ann. Biomed. Eng., vol. 31, no. 7, pp. 753-760, Jul. 2003.

[39] H. A. Leddy, M. A. Haider, and F. Guilak, "Diffusional Anisotropy in Collagenous Tissues: Fluorescence Imaging of Continuous Point Photobleaching," Biophys. J., vol. 91, no. 1, pp. 311-316, Jul. 2006.

[40] C. D. DiDomenico, A. Kaghazchi, and L. J. Bonassar, "Measurement of local diffusion and composition in degraded articular cartilage reveals the unique role of surface structure in controlling macromolecular transport," J. Biomech., vol. 82, no. October, pp. 38-45, 2019.

[41] B. T. Graham, A. C. Moore, D. L. Burris, and C. Price, "Sliding enhances fluid and solute transport into buried articular cartilage contacts," Osteoarthr. Cartil., vol. 25, no. 12, pp. 2100-2107, 2017.

[42] A. C. Moore and D. L. Burris, "Tribological rehydration of cartilage and its potential role 
in preserving joint health," Osteoarthr. Cartil., vol. 25, no. 1, pp. 99-107, 2017.

[43] P. T. Williams, "Effects of Running and Walking on Osteoarthritis and Hip Replacement Risk," Med Sci Sport. Exerc, vol. 45, no. 7, pp. 1292-1297, 2013.

[44] B. P. O'Hara, J. P. Urban, and A. Maroudas, "Influence of cyclic loading on the nutrition of articular cartilage.," Ann. Rheum. Dis., vol. 49, no. 7, pp. 536-9, Jul. 1990.

[45] C. D. Di Domenico, Z. X. Wang, and L. J. Bonassar, "Cyclic mechanical loading enhances transport of antibodies into articular cartilage," J. Biomech. Eng., vol. 139, no. 1, 2017.

[46] C. D. DiDomenico and L. J. Bonassar, "The Effect of Charge and Mechanical Loading on Antibody Diffusion Through the Articular Surface of Cartilage," J. Biomech. Eng., vol. 141, no. 1, pp. 1-6, 2019.

[47] H. Meng et al., "Diffusion of neutral solutes within human osteoarthritic cartilage: Effect of loading patterns," J. Orthop. Transl., vol. 22, pp. 58-66, 2020.

[48] B. T. Graham, A. C. Moore, D. L. Burris, and C. Price, "Mapping the spatiotemporal evolution of solute transport in articular cartilage explants reveals how cartilage recovers fluid within the contact area during sliding.," J. Biomech., vol. 71, pp. 271-276, 2018.

[49] R. C. Evans and T. M. Quinn, "Dynamic compression augments interstitial transport of a glucose-like solute in articular cartilage.," Biophys. J., vol. 91, no. 4, pp. 1541-7, Aug. 2006.

[50] P. A. Torzilli, T. C. Adams, and R. J. Mis, "Transient solute diffusion in articular cartilage," J. Biomech., vol. 20, no. 2, pp. 203-214, 1987.

[51] V. C. Mow, A. Ratcliffe, and A. Robin Poole, "Cartilage and diarthrodial joints as paradigms for hierarchical materials and structures," Biomaterials, vol. 13, no. 2, pp. 6797, Jan. 1992.

[52] J. Crank, The Mathematics of Diffusion, 2nd ed. Uxbridge: Clarendon Press, 1975.

[53] E. J. Carr and I. W. Turner, "A semi-analytical solution for multilayer diffusion in a composite medium consisting of a large number of layers," Appl. Math. Model., vol. 40, no. 15-16, pp. 7034-7050, 2016.

[54] R. C. Evans and T. M. Quinn, "Solute convection in dynamically compressed cartilage," J. Biomech., vol. 39, no. 6, pp. 1048-1055, 2006.

[55] S. Zhou, Z. Cui, and J. P. G. Urban, "Factors influencing the oxygen concentration gradient from the synovial surface of articular cartilage to the cartilage-bone interface: A modeling study," Arthritis Rheum., vol. 50, no. 12, pp. 3915-3924, Dec. 2004.

[56] A. Maroudas, M. T. Bayliss, N. Uchitel-Kaushansky, R. Schneiderman, and E. Gilav, "Aggrecan turnover in human articular cartilage: use of aspartic acid racemization as a marker of molecular age.," Arch. Biochem. Biophys., vol. 350, no. 1, pp. 61-71, Feb. 1998. 
[57] P. A. Torzilli, D. A. Dethmers, D. E. Rose, and H. F. Schryuer, "Movement of interstitial water through loaded articular cartilage," J. Biomech., vol. 16, no. 3, 1983.

[58] A. R. Gannon, T. Nagel, and D. J. Kelly, "The role of the superficial region in determining the dynamic properties of articular cartilage," Osteoarthr. Cartil., vol. 20, no. 11, pp. 1417-1425, 2012.

[59] A. R. C. Jones et al., "Binding and localization of recombinant Lubricin to articular cartilage surfaces," J. Orthop. Res., vol. 25, no. 3, pp. 283-292, Mar. 2007.

[60] T. C. Pataky, "Generalized n-dimensional biomechanical field analysis using statistical parametric mapping,” J. Biomech., vol. 43, no. 10, pp. 1976-1982, 2010.

[61] A. D. Speirs and A. Shegaf, "A More Physiological Loading Mode for Cartilage Mechanobiological Testing," in ORS Annual Meeting, 2020.

[62] A. Shegaf and A. Speirs, "Cartilage Biomechanical Response Differs Under Physiological Biaxial Loads and Uniaxial Cyclic Compression," J. Biomech. Eng., vol. 142, no. 5, pp. $1-5,2020$.

[63] M. B. Albro et al., "Raman spectroscopic imaging for quantification of depth-dependent and local heterogeneities in native and engineered cartilage," npj Regen. Med., vol. 3, no. 1, p. 3, Dec. 2018.

[64] M. B. Albro et al., "Heterogeneous engineered cartilage growth results from gradients of media-supplemented active TGF- $\beta$ and is ameliorated by the alternative supplementation of latent TGF- $\beta$.," Biomaterials, vol. 77, pp. 173-185, Jan. 2016.

[65] A. D. Cigan, R. J. Nims, M. B. Albro, G. Vunjak-Novakovic, C. T. Hung, and G. A. Ateshian, "Nutrient channels and stirring enhanced the composition and stiffness of large cartilage constructs.," J. Biomech., vol. 47, no. 16, pp. 3847-54, Dec. 2014.

[66] C. D. DiDomenico and L. J. Bonassar, "How can 50 years of solute transport data in articular cartilage inform the design of arthritis therapeutics?," Osteoarthr. Cartil., vol. 26, no. 11, pp. 1438-1446, 2018.

[67] C. Larsen et al., "Intra-articular depot formulation principles: Role in the management of postoperative pain and arthritic disorders," J. Pharm. Sci., vol. 97, no. 11, pp. 4622-4654, Nov. 2008.

[68] L. J. Bonassar, A. J. Grodzinsky, E. H. Frank, S. G. Davila, N. R. Bhaktav, and S. B. Trippel, "The effect of dynamic compression on the response of articular cartilage to insulin-like growth factor-I," J. Orthop. Res., vol. 19, no. 1, pp. 11-17, Jan. 2001.

[69] K. P. Arkill and C. P. Winlove, "Solute transport in the deep and calcified zones of articular cartilage," Osteoarthr. Cartil., vol. 16, no. 6, pp. 708-714, 2008.

[70] G. W. Recktenwald, "Finite-Difference Approximations to the Heat Equation," 2004.

[71] I. Martin, B. Obradovic, L. E. Freed, and G. Vunjak-Novakovic, "Method for Quantitative Analysis of Glycosaminoglycan Distribution in Cultured Natural and Engineered Cartilage," Ann. Biomed. Eng., vol. 27, no. 5, pp. 656-662, 1999. 
[72] K. P. H. Pritzker et al., "Osteoarthritis cartilage histopathology: Grading and staging," Osteoarthr. Cartil., vol. 14, no. 1, pp. 13-29, 2006.

[73] S. Milne et al., "Continuous passive motion following total knee arthroplasty," in Cochrane Database of Systematic Reviews, no. 2, John Wiley \& Sons, Ltd, 2003.

[74] D. M. Knapik et al., "The basic science of continuous passive motion in promoting knee health: A systematic review of studies in a rabbit model," Arthroscopy - Journal of Arthroscopic and Related Surgery, vol. 29, no. 10. pp. 1722-1731, Oct-2013.

[75] S. W. O'Driscoll, F. W. Keeley, and R. B. Salter, "Durability of regenerated articular cartilage produced by free autogenous periosteal grafts in major full-thickness defects in joint surfaces under the influence of continuous passive motion. A follow-up report at one year," J. Bone Jt. Surg. - Ser. A, vol. 70, no. 4, pp. 595-606, Apr. 1988.

[76] J. S. Howard, C. G. Mattacola, S. E. Romine, and C. Lattermann, "Continuous Passive Motion, Early Weight Bearing, and Active Motion following Knee Articular Cartilage Repair: Evidence for Clinical Practice.," Cartilage, vol. 1, no. 4, pp. 276-86, Oct. 2010.

[77] D. Zhao, S. A. Banks, D. D. D’Lima, C. W. Colwell, and B. J. Fregly, "In vivo medial and lateral tibial loads during dynamic and high flexion activities," J. Orthop. Res., vol. 25, no. 5, pp. 593-602, May 2007.

[78] G. Bergmann et al., "Hip contact forces and gait patterns from routine activities," in Journal of Biomechanics, 2001, vol. 34, no. 7, pp. 859-871.

[79] P. A. Torzilli, "Effects of temperature, concentration and articular surface removal on transient solute diffusion in articular cartilage," Med. Biol. Eng. Comput., vol. 31, no. 1, pp. S93-S98, Jul. 1993.

[80] A. E. Peters, E. J. Comerford, S. Macaulay, K. T. Bates, and R. Akhtar, "Micromechanical properties of canine femoral articular cartilage following multiple freeze-thaw cycles," $J$. Mech. Behav. Biomed. Mater., vol. 71, pp. 114-121, Jul. 2017.

[81] C. Qu, M. Hirviniemi, V. Tiitu, J. S. Jurvelin, J. Töyräs, and M. J. Lammi, "Effects of Freeze-Thaw Cycle with and without Proteolysis Inhibitors and Cryopreservant on the Biochemical and Biomechanical Properties of Articular Cartilage," Cartilage, vol. 5, no. 2, pp. 97-106, Apr. 2014.

[82] A. Changoor, L. Fereydoonzad, A. Yaroshinsky, and M. D. Buschmann, "Effects of Refrigeration and Freezing on the Electromechanical and Biomechanical Properties of Articular Cartilage," J. Biomech. Eng., vol. 132, no. 6, Jun. 2010.

[83] K. Tavakol, R. G. Miller, D. P. Bazett-Jones, W. S. Hwang, L. E. McGann, and N. S. Schachar, "Ultrastructural changes of articular cartilage chondrocytes associated with freeze-thawing," J. Orthop. Res., vol. 11, no. 1, pp. 1-9, 1993.

[84] B. S. Gardiner, L. Zhang, D. W. Smith, P. Pivonka, and A. J. Grodzinsky, “A mathematical model for targeting chemicals to tissues by exploiting complex degradation," Biol. Direct, vol. 6, no. 1, p. 46, 2011. 
[85] X. Chen, O. Nadiarynkh, S. Plotnikov, and P. J. Campagnola, "Second harmonic generation microscopy for quantitative analysis of collagen fibrillar structure," Nat. Protoc., vol. 7, no. 4, pp. 654-669, Apr. 2012.

[86] M. Ferretti et al., "Anti-inflammatory effects of continuous passive motion on meniscal fibrocartilage," J. Orthop. Res., vol. 23, no. 5, pp. 1165-1171, Sep. 2005. 


\section{Appendix A: Conversion from fluorescent intensity to concentration}


Serial dilutions were generated from the stock solution of Na-fluorescein $(0.01 \mathrm{mg} / \mathrm{ml}$ prepared using PBS, $\mathrm{pH}$ 7.0). A total of 13 dilutions were generated ranging from $0.0001-0.004 \mathrm{mg} / \mathrm{ml}$. Solutions were imaged in capillary tubes under the same microscope settings used in the experimental protocol. A calibration curve was generated by plotting the pixel intensities against the corresponding concentrations. The relationship between concentration and pixel intensity was linear in the ranges used for this study $\left(\mathrm{R}^{2}=0.9968\right)$.

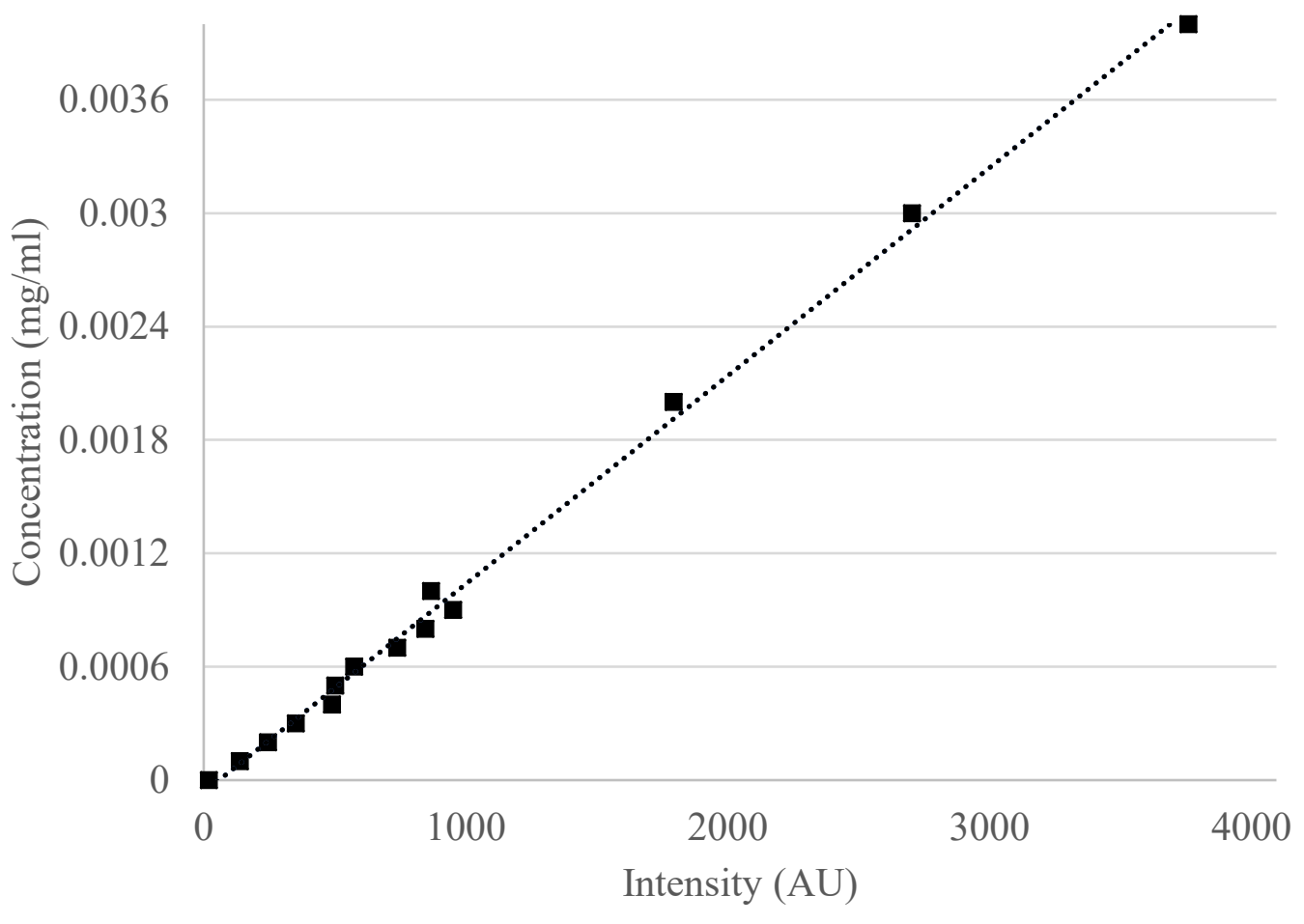

Figure A-1: Calibration curve generated for converting fluorescent intensity to concentration. 
Appendix B: Results of solute transport following

lubricin removal 


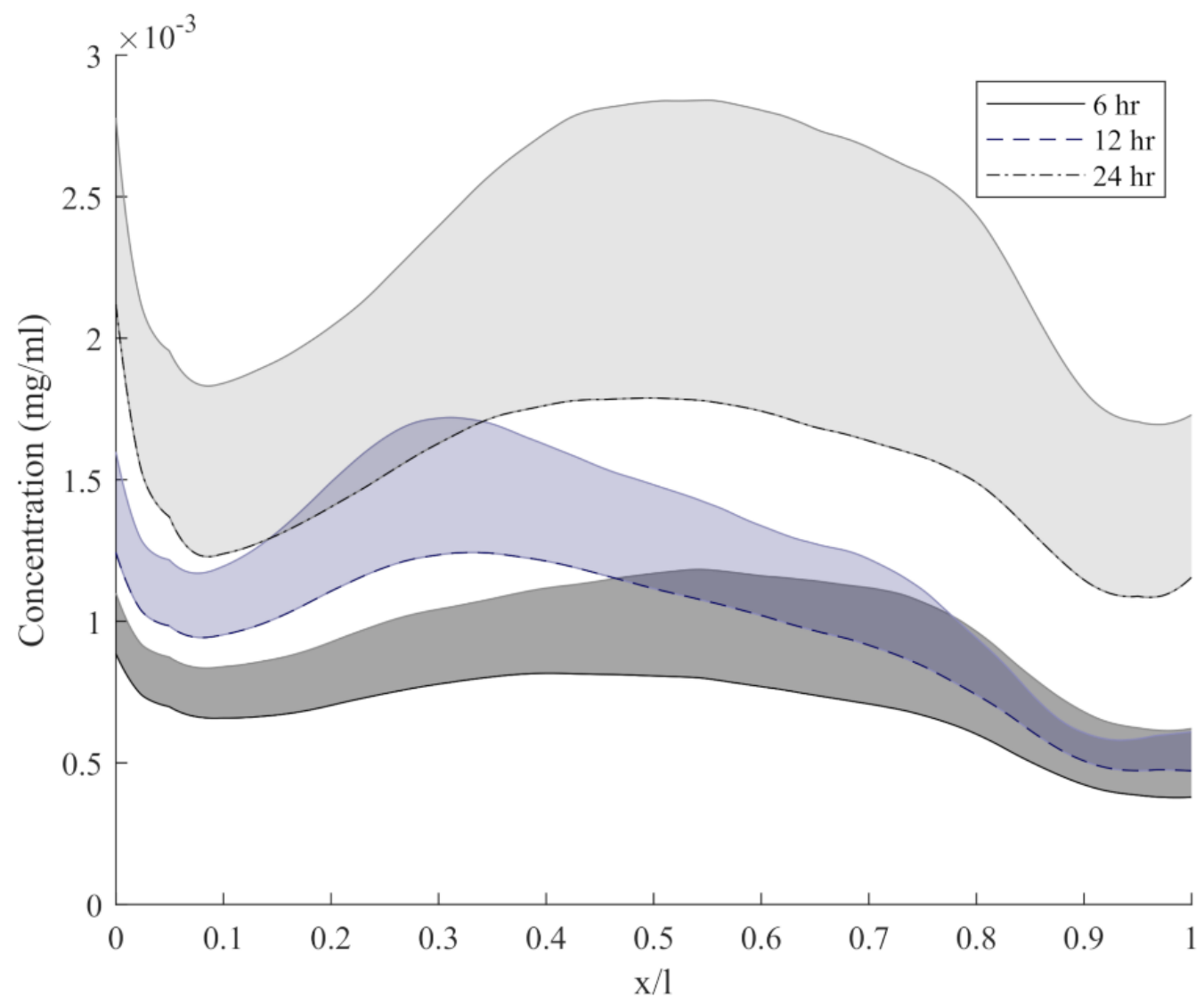

Figure B-1: Mean concentration profiles for specimens subject to the lubricin extraction protocol. Shaded region shows $+95 \%$ confidence interval of the mean. Samples sizes were 7, 6, and 6 for $6 \mathrm{hr}$, $12 \mathrm{hr}$, and $24 \mathrm{hr}$ timepoints respectively. 

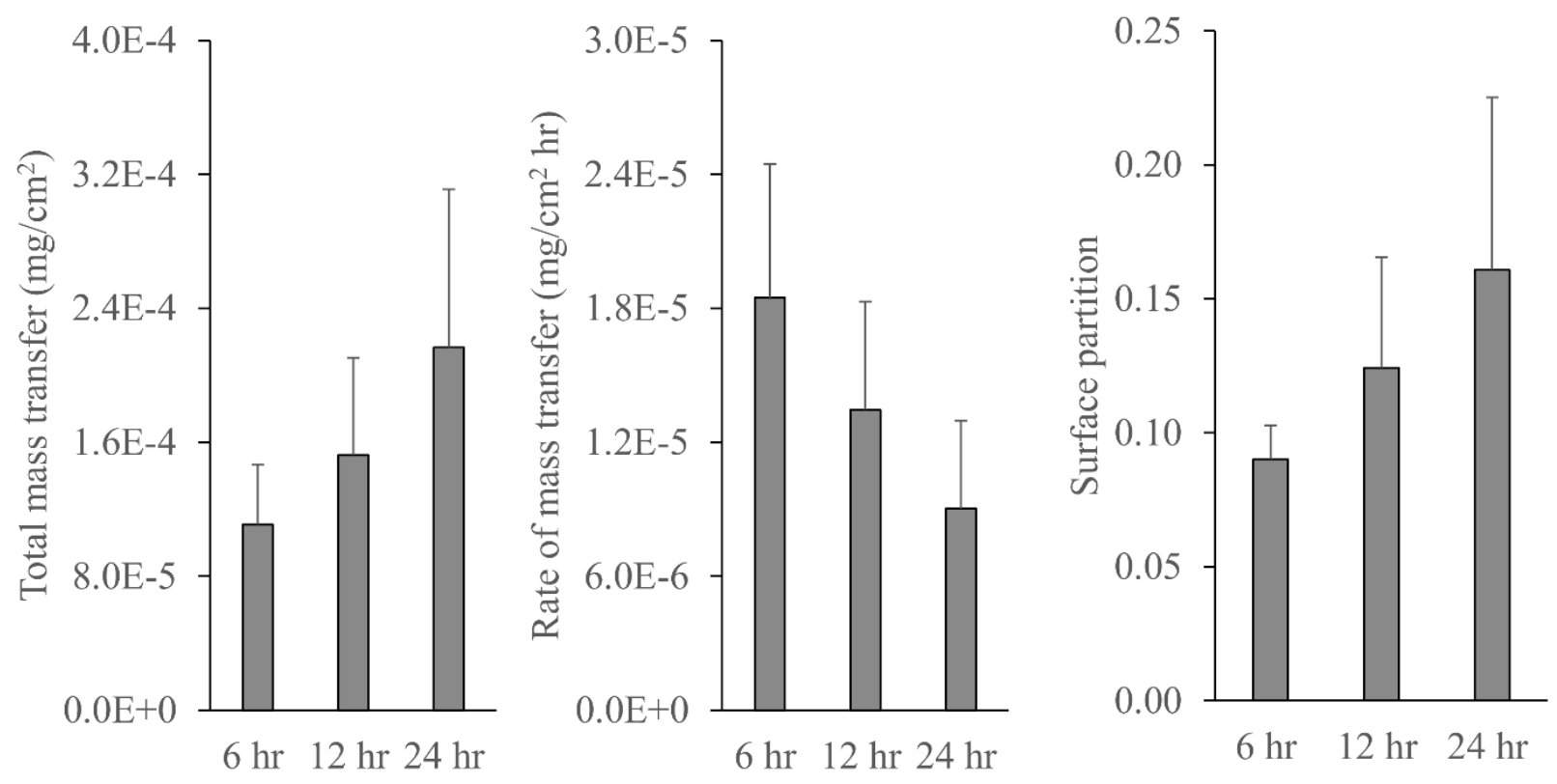

Figure B-2: Mean mass transfer, rate of mass transfer and surface partition factor for specimens subject to lubricin extraction protocol. Error bar shows $+95 \%$ confidence interval of the mean. 


\section{Appendix C: Multilayer model sensitivity analysis to determine number of layers}




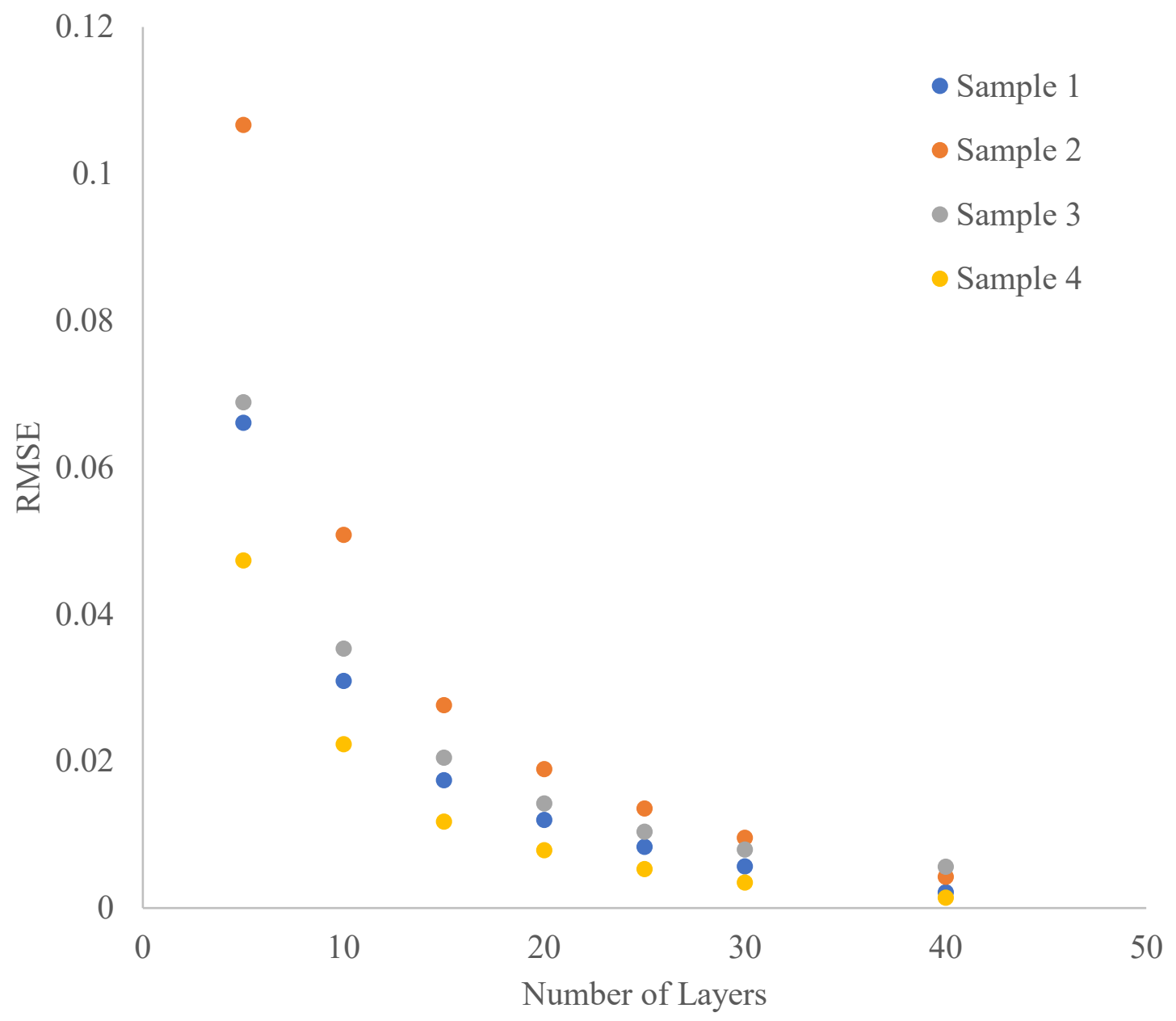

Figure C-1: Sensitivity analysis for determining the number of subdivisions within the multilayer model. 20 layers were chosen as a compromise between accuracy and computational time. Results shown for 4 randomly selected samples. 


\section{Appendix D: Single layer and multilayer model fits to all specimens}




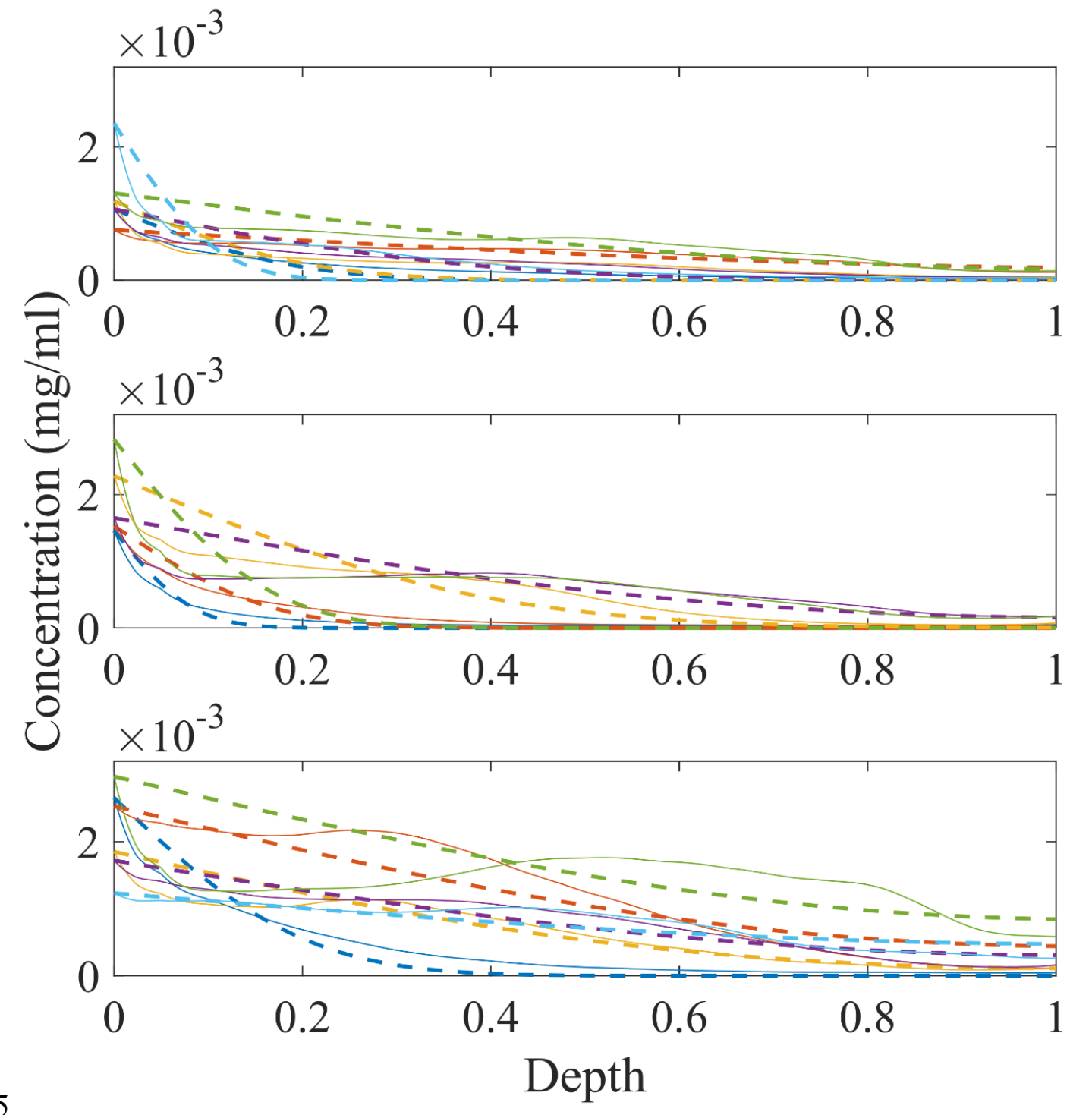

Figure D-1: Single layer model fits to intensity profiles for samples subject to sliding. Dashed line indicates model fit, solid line represents experimental data. Experimental time points 0.5 hr (top), 1 hr (middle), and 2 hr (bottom). 


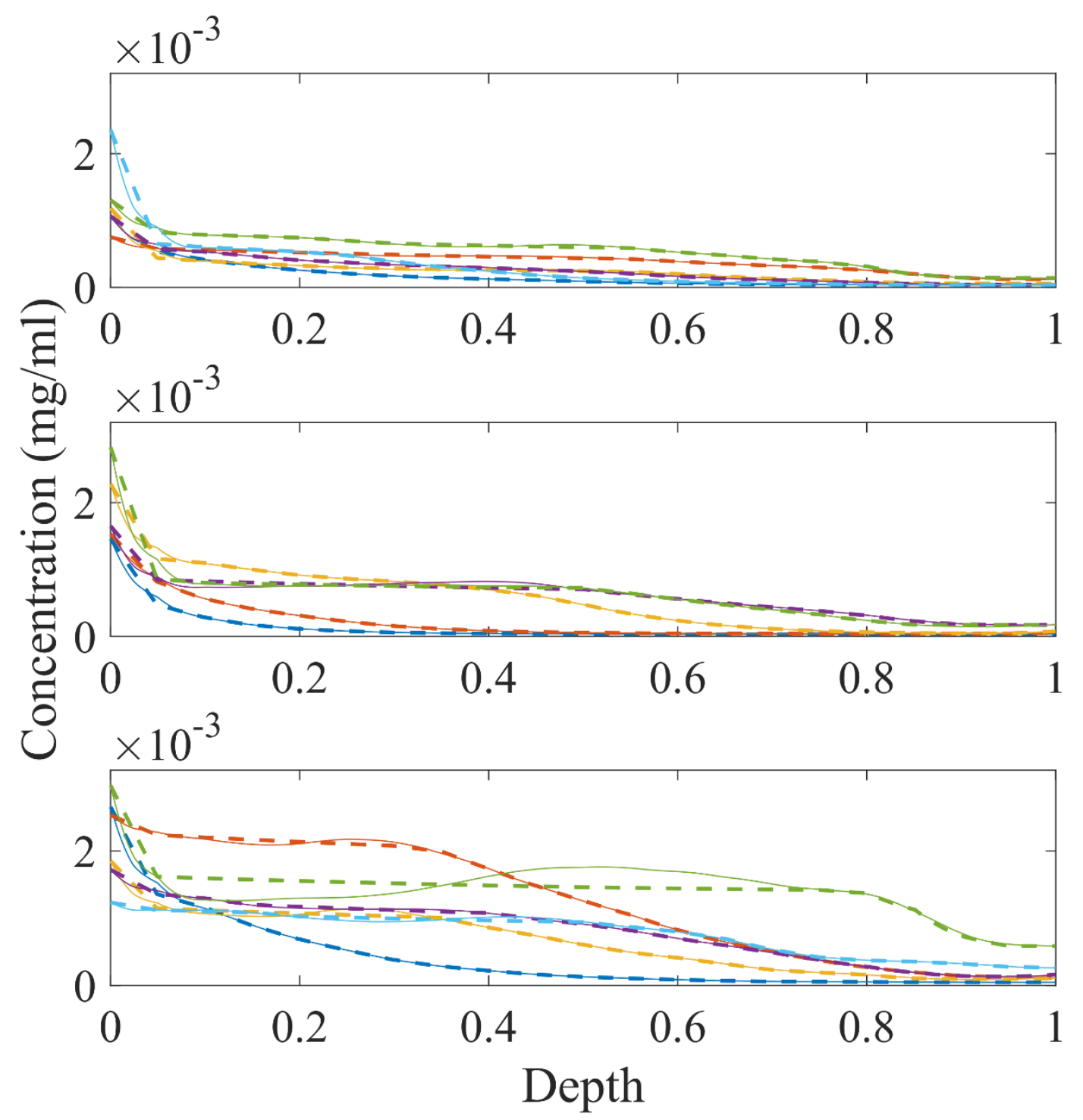

Figure D-2: Multilayer model fits to intensity profiles for samples subject to sliding. Dashed line indicates model fit, solid line represents experimental data. Experimental time points $0.5 \mathrm{hr}$ (top), 1 hr (middle), and 2 hr (bottom). 




Figure D-3: Single layer model fits to intensity profiles for samples subject to uniaxial compression. Dashed line indicates model fit, solid line represents experimental data. Experimental time points $0.5 \mathrm{hr}$ (top), $1 \mathrm{hr}$ (middle), and $2 \mathrm{hr}$ (bottom). 


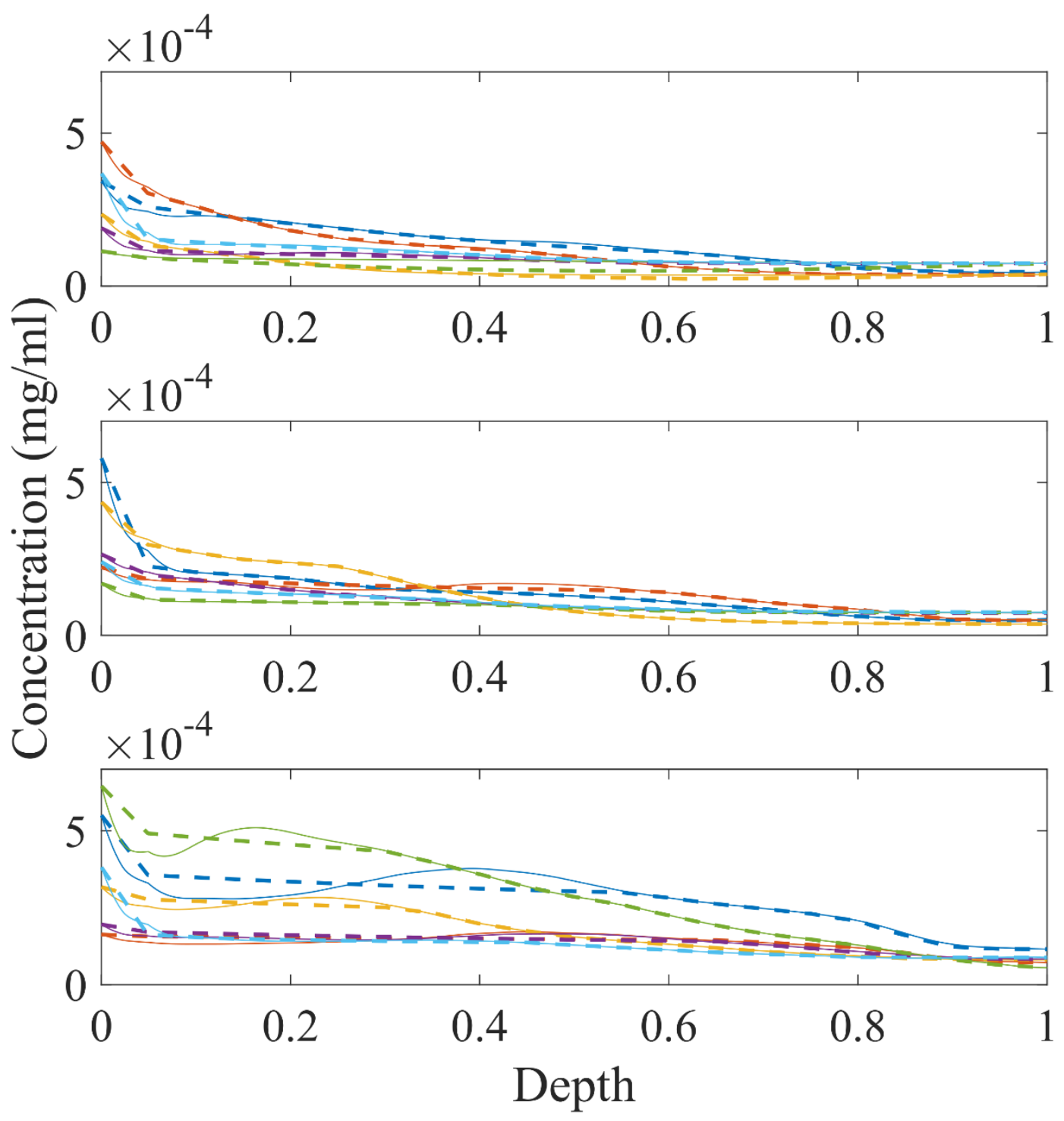

Figure D-4: Multilayer model fits to intensity profiles for samples subject to uniaxial compression. Dashed line indicates model fit, solid line represents experimental data. Experimental time points 0.5 hr (top), 1 hr (middle), and 2 hr (bottom). 